\title{
WIYN Open Cluster Study. LXXVI. Li Evolution Among Stars of Low/Intermediate Mass: The Metal-deficient Open Cluster NGC 2506
}

\author{
Barbara J. Anthony-Twarog ${ }^{1}$ (D), Donald B. Lee-Brown ${ }^{1}$ (D), Constantine P. Deliyannis ${ }^{2}$, and Bruce A. Twarog ${ }^{1}$ \\ ${ }^{1}$ Department of Physics and Astronomy, University of Kansas, Lawrence, KS 66045-7582, USA; bjat@ku.edu, donald@ku.edu, btwarog@ku.edu \\ 2 Department of Astronomy, Indiana University, Bloomington, IN 47405-7105, USA; cdeliyan@indiana.edu \\ Received 2017 December 8; revised 2018 February 1; accepted 2018 February 1; published 2018 March 6
}

\begin{abstract}
HYDRA spectra of 287 stars in the field of NGC 2506 from the turnoff through the giant branch are analyzed. With previous data, 22 are identified as probable binaries; 90 more are classified as potential non-members. Spectroscopic analyses of $\sim 60$ red giants and slowly rotating turnoff stars using line equivalent widths and a neural network approach lead to $[\mathrm{Fe} / \mathrm{H}]=-0.27 \pm 0.07$ (s.d.) and $[\mathrm{Fe} / \mathrm{H}]=-0.27 \pm 0.06$ (s.d.), respectively. Li abundances are derived for 145 probable single-star members, 44 being upper limits. Among turnoff stars outside the Li-dip, $\mathrm{A}(\mathrm{Li})=3.04 \pm 0.16$ (s.d.), with no trend with color, luminosity, or rotation speed. Evolving from the turnoff across the subgiant branch, there is a well-delineated decline to $\mathrm{A}(\mathrm{Li})$ $\sim 1.25$ at the giant branch base, coupled with the rotational spindown from between $\sim 20$ and $70 \mathrm{~km} \mathrm{~s}^{-1}$ to less than $20 \mathrm{~km} \mathrm{~s}^{-1}$ for stars entering the subgiant branch and beyond. A(Li) remains effectively constant from the giant branch base to the red giant clump level. A new member above the clump redefines the path of the firstascent red giant branch; its $\mathrm{Li}$ is 0.6 dex below the first-ascent red giants. With one exception, all post-He-flash stars have upper limits to $\mathrm{A}(\mathrm{Li})$, at or below the level of the brightest first-ascent red giant. The patterns are in excellent qualitative agreement with the model predictions for low/intermediate-mass stars which undergo rotation-induced mixing at the turnoff and subgiant branch, first dredge-up, and thermohaline mixing beyond the red giant bump.
\end{abstract}

Key words: open clusters and associations: individual (NGC 2506) - stars: abundances - stars: evolution

Supporting material: machine-readable tables

\section{Introduction}

This paper presents a spectroscopic complement to the intermediate-band photometric analysis of the old open cluster, NGC 2506 (Anthony-Twarog et al. 2016, hereinafter AT16). The background history of this cluster is explored in detail in AT16 and will not be repeated here except when required by the specifics of the analysis. Suffice it to say that precision photometry on the extended Strömgren system $(u v b y C a \mathrm{H} \beta)$ has established that the cluster is subject to low reddening $(E(B-V)=0.058 \pm 0.001)$ with negligible variation across its face (AT16). More important for our purposes, as derived from two photometric indices, the cluster is moderately metalpoor $([\mathrm{Fe} / \mathrm{H}]=-0.32 \pm 0.03)$ relative to the typical cluster in the solar neighborhood, a not unexpected result for a cluster positioned beyond the solar circle in the anticenter region (e.g., Twarog et al. 1997; Friel et al. 2002; Netopil et al. 2016). The key element, however, enhancing its role is its age, now well established at $1.85 \pm 0.05 \mathrm{Gyr}$ based upon application of the Victoria-Regina (VR) isochrones (VandenBerg et al. 2006) on the Strömgren system. The revised cluster age places NGC 2506 in a category comparable to NGC 3680 at $1.7 \mathrm{Gyr}$ (Anthony-Twarog et al. 2009), but slightly older than NGC 752 at $1.45 \mathrm{Gyr}$ (Twarog et al. 2015) and IC 4651 at $1.5 \mathrm{Gyr}$ (Anthony-Twarog et al. 2009); these clusters cover a range in metallicity of $\sim 0.45 \mathrm{dex}$ in $[\mathrm{Fe} / \mathrm{H}]$.

Since the overarching goal of our cluster program is to evaluate and understand the role of mixing/convection in the atmospheres and interiors of stars of low to intermediate mass both on and off the main sequence, NGC 2506 holds a pivotal position between the younger clusters like NGC 7789 (1.4 Gyr) and NGC 752 and the clearly older example of NGC 6819
(2.25 Gyr) (C. P. Deliyannis et al. 2018, in preparation). The slightly lower turnoff mass of NGC 2506 due to the higher age and lower $[\mathrm{Fe} / \mathrm{H}]$, coupled with the richness of the cluster, allows delineation of the subgiant branch between the turnoff and the first-ascent red giant (FRG) branch below the red giant clump to a degree previously impossible, even with the combined samples of NGC 3680, NGC 752, and IC 4651 (Anthony-Twarog et al. 2009).

The outline of the paper is as follows: Section 2 discusses the compilation of potential spectroscopic candidates within NGC 2506, with special emphasis on the subgiant branch and stars fainter than the red giant clump, and the HYDRA observations that form the core of this study; Section 3 uses radial velocities and proper motions, when available, to identify and isolate the most probable members, whether single stars or binaries, and to eliminate likely field stars from the analysis. Section 4 lays out the metallicity derivation of the cluster for key elements adopting a traditional technique based upon the equivalent widths (EWs) of lines in a modest wavelength region centered on the $\mathrm{Li} 6708 \mathrm{~A}$ line, as well as a new approach for temperature and metallicity estimation built around a neural network. Section 5 contains the derivation of the $\mathrm{Li}$ abundance and demonstrates the critical place of NGC 2506 in probing the role of mixing/convection for stars evolving from the main sequence to the giant branch. Section 6 is a summary of our conclusions.

\section{Observations and Data Reduction}

\subsection{Sample Selection}

The starting point for the selection of potential cluster members is the proper-motion study of Chiu \& van Altena 
(1981). There is little doubt that the stars defined as $70 \%$ or higher probable members based upon proper motion alone form a well-defined sample which nicely delineates the primary cluster sequences in both broad-band (McClure et al. 1981; Marconi et al. 1997; Kim et al. 2001) and intermediate-band (AT16) photometry. The weaknesses in relying solely upon this selection criterion are apparent: (a) even with a rather high proper-motion probability cutoff of $70 \%$, field star contamination can occur in the absence of the third vector component, the radial velocity; (b) proper-motion precision declines with increasing $V$, leading to the elimination of a higher fraction of true members and contamination by more non-members at the turnoff and fainter; and (c) the proper-motion and original broad-band surveys extend radially to only $\sim 5^{\prime}$ from the cluster center. Though the dominant majority of the cluster members should be contained within this zone (Lee et al. 2013), the desire to populate and survey the color-magnitude diagram (CMD) as completely as possible, especially along some of the relatively rapid phases of evolution beyond the the main sequence, requires a broader search to identify as many NGC 2506 inhabitants as possible.

\subsection{Photometric Input}

To expand the sample, use was made of two CCD surveys covering comparable fields-of-view, i.e., $20^{\prime}$ on a side. Three broad-band CCD surveys of NGC 2506 have been undertaken to date by Marconi et al. (1997), Kim et al. (2001), and Lee et al. (2012); the surveys cover too small an area, suffer from larger than acceptable photometric scatter, or remain unpublished, respectively. The broad-band data for the current discussion (Van Stockum et al. 2005) were obtained on 2004 November 6 with the S2KB CCD at the $f / 7.5$ focus of the WIYN $0.9 \mathrm{~m}$ telescope at Kitt Peak National Observatory, with a scale of 0 ". 60 per pixel, and seeing in the range 1 !" $3-1$ ". 6 . Cluster images were reduced using DAOPHOT II (Stetson 1987). Typically, a few hundred bright, isolated stars were used to determine a point-spread function, which was allowed to vary spatially, and a subsequent small (typically 0.01-0.02 mag) aperture correction, which was also allowed to vary spatially. After rejection of outliers, approximately 50 Landolt (1992) standards for each filter provided a calibration onto the Johnson-Cousins-Landolt system, and verified the photometricity of the night, as defined by zero-point errors in the transformations below $0.01 \mathrm{mag}$. The intermediate-band photometry (AT16) came from the $4000 \times 4000$ CCD camera ${ }^{3}$ on the $1.0 \mathrm{~m}$ telescope operated by the $\mathrm{SMARTS}^{4}$ consortium at Cerro Tololo Inter-American Observatory. All stars with membership probabilities greater than $90 \%$ were used to define mean relations from the main sequence at $V$ brighter than 15.6 to the top of the giant branch. Stars whose broad-band photometric errors placed them within the range of the mean relations and had proper-motion probabilities above $50 \%$ were identified and initially selected. For stars outside the spatial range of the astrometric survey, location within range of the CMD relations provided the sole criterion for potential followup observations.

\footnotetext{
3 http://www.astronomy.ohio-state.edu/Y4KCam/detector

4 http://www.astro.yale.edu/smarts
}

\subsection{Spectroscopic Observations}

Spectroscopic data were obtained using the WIYN $3.5 \mathrm{~m}$ telescope ${ }^{5}$ and the HYDRA multi-object spectrograph over 19 nights from 2015 January $16 / 17$ to 2017 February $24 / 25$. Nine configurations were designed to position fibers on a total of 287 stars, with 38 stars observed in more than one fiber configuration. Individual exposures ranged from 10 to 90 minutes, with accumulated totals of two hours for stars in the brightest two configurations targeting red giants, 5-7.5 hr for the four configurations aimed at subgiant stars, and between 9 and $12.5 \mathrm{hr}$ total for three constructed to sample the turnoff region and upper main sequence of the cluster.

In these $58 \mathrm{hr}$ of observing over a 25 month period, we obtained spectra with signal-to-noise per pixel $\geqslant 100$ for all 287 stars. Our spectra cover a wavelength range $\sim 400 \AA$ wide centered on $6650 \AA$ with per-pixel resolution of $0.2 \AA$ and a resolution of $R \sim 13,000$. Details on the reduction procedure can be found in Lee-Brown et al. (2015) and will not be repeated here.

Multiple exposures of any particular fiber configuration were combined if the observations were obtained within the same run of a few adjacent nights. A few of our configurations were observed over a period of a year or more, with some fiber losses in the interim. In these cases, the combination of individual spectra for stars obtained through different fibers or in different years was accomplished by undoing the individual throughput corrections before combining the spectra. This was also the procedure for stars observed in different runs as part of different fiber configurations. Combination of spectra from widely separated epochs was only carried out if there appeared to be no significant velocity shift.

As always, inclusion of specific stars within the spectroscopic survey was ultimately constrained by the need to optimize HYDRA configurations over multiple observing runs. As preliminary reductions revealed that some stars were clear radial-velocity non-members, these were dropped from the program and others, usually potential subgiants and giants, were added. A small set of definite non-members was included in the database to use as a control for comparison with the hopefully homogeneous group of metal-deficient cluster members.

Before discussing the results for individual stars, it should be noted that, whenever available, a star will be referred to using its WEBDA identification. However, since the sample here covers a much broader area than that of past published surveys, regrettably another identification number must be added for those stars outside the area listed within WEBDA. Column 1 of Table 1 lists WEBDA identification numbers for stars; numbers greater than 7000 refer to stars added by this survey. Table 1 lists stars in sequence based upon their right ascension; coordinates given in Table 1 are identical to those found in AT16 for all but 10 stars not included in the photometric study. The portion of Table 1 here includes enough lines to show the form and content of the larger table available online.

A point of confusion regarding the WEBDA identifications does require correction. In the original photometric and astrometric surveys (Chiu \& van Altena 1981; McClure et al. 1981), the stars were numbered using a quadrant number,

\footnotetext{
5 The WIYN Observatory was a joint facility of the University of WisconsinMadison, Indiana University, Yale University, and the National Optical Astronomy Observatory.
} 
Table 1

Velocity and Membership Information for Spectroscopic Sample in NGC 2506

\begin{tabular}{|c|c|c|c|c|c|c|c|c|c|c|}
\hline ID No. & $\alpha(2000)$ & $\delta(2000)$ & $\operatorname{Prob}(\mu)$ & $V_{\text {rad }}$ & $\sigma\left(V_{\mathrm{rad}}\right)$ & $V_{\mathrm{rot}} \sin i$ & $\sigma\left(V_{\mathrm{rot}} \sin i\right)$ & $(B-V)$ & $V$ & Membership \\
\hline 7001 & 119.838542 & -10.705722 & $\cdots$ & 50.1 & 0.8 & 18.0 & 0.4 & 0.886 & 14.990 & NM \\
\hline 7002 & 119.839917 & -10.872389 & $\cdots$ & 44.5 & 0.8 & 19.9 & 0.5 & 0.988 & 12.494 & $\mathrm{NM}$ \\
\hline 7003 & 119.842792 & -10.805722 & $\ldots$ & -23.5 & 1.5 & 28.6 & 1.2 & 0.464 & 14.859 & $\mathrm{NM}$ \\
\hline 7004 & 119.843833 & -10.855722 & $\ldots$ & 61.0 & 0.7 & 17.3 & 0.4 & 0.856 & 14.578 & NM \\
\hline 7005 & 119.845708 & -10.889056 & $\ldots$ & 60.6 & 0.1 & 16.2 & 1.7 & 1.030 & 13.466 & $\mathrm{NM}$ \\
\hline 7006 & 119.850667 & -10.822389 & $\ldots$ & 27.0 & 2.6 & 33.2 & 2.0 & 0.322 & 14.689 & NM \\
\hline 7007 & 119.852095 & -10.891248 & $\cdots$ & -13.5 & 1.6 & 51.1 & 1.3 & 0.890 & 11.600 & NM \\
\hline 7008 & 119.852125 & -10.839056 & $\cdots$ & 83.6 & 0.7 & 9.1 & 0.2 & 0.897 & 14.703 & M \\
\hline 7009 & 119.855000 & -10.789056 & $\ldots$ & 74.6 & 0.7 & 20.3 & 0.5 & 0.642 & 14.495 & NM \\
\hline 7010 & 119.857170 & -10.924294 & $\ldots$ & 103.7 & 2.3 & 29.2 & 2.7 & 0.394 & 15.210 & NM \\
\hline 7011 & 119.861862 & -10.760853 & $\cdots$ & 101.5 & 0.7 & 11.0 & 0.3 & 1.071 & 13.935 & $\mathrm{NM}$ \\
\hline 7012 & 119.862495 & -10.905938 & $\ldots$ & 69.4 & 0.7 & 11.3 & 0.3 & 1.061 & 13.904 & NM \\
\hline 7013 & 119.862595 & -10.620172 & $\cdots$ & 39.3 & 1.1 & 18.2 & 0.6 & 1.304 & 12.546 & NM \\
\hline 7014 & 119.864540 & -10.904671 & $\cdots$ & 23.1 & 0.6 & 9.7 & 0.2 & 0.600 & 14.529 & $\mathrm{NM}$ \\
\hline 7015 & 119.866119 & -10.894622 & $\cdots$ & 83.8 & 2.7 & 85.5 & 6.6 & 0.399 & 15.174 & M \\
\hline 7016 & 119.871458 & -10.939056 & $\ldots$ & -45.9 & 1.0 & 14.2 & 0.5 & 0.575 & 14.335 & NM \\
\hline 7017 & 119.872849 & -10.654552 & $\cdots$ & 59.8 & 2.4 & 60.7 & 3.3 & 0.439 & 14.419 & NM \\
\hline 7018 & 119.879631 & -10.691129 & $\cdots$ & 27.0 & 0.5 & 13.7 & 0.2 & 0.873 & 12.398 & NM \\
\hline 7019 & 119.880058 & -10.631248 & $\cdots$ & 82.9 & 0.6 & 9.1 & 0.2 & 0.910 & 14.548 & M \\
\hline 7020 & 119.880890 & -10.916945 & $\ldots$ & 20.1 & 0.6 & 14.8 & 0.3 & 0.782 & 14.730 & $\mathrm{NM}$ \\
\hline
\end{tabular}

(This table is available in its entirety in machine-readable form.)

ring number, and sequential count within that zone. For quadrant 2 , ring 3 , the number of stars extended to 106 , so the last star measured was 23106. WEBDA incorrectly rewrote the numbers above 100 as ring 4, i.e., 23106 became 2406. This leads to confusion because in quadrants 2 and 4, there are photoelectric standards outside the three rings of the photographic survey numbered 2401, 2402, 4401, and 4402. WEBDA 2401 and 2402 are actually 23101 and 23102. In our table and analysis, 2401 and 2402 refer to the original designations as marked on the cluster chart (Figure 1) of McClure et al. (1981).

\subsection{Radial Velocities and Rotation}

Individual heliocentric stellar radial velocities, $V_{\text {rad }}$, were derived from each summed composite spectrum utilizing the Fourier-transform, cross-correlation facility fxcor in IRAF. ${ }^{6}$ In this utility, program stars are compared to stellar templates of similar effective temperature $\left(T_{\text {eff }}\right)$ over the full wavelength range of our spectra excluding the immediate vicinity of the $\mathrm{H} \alpha$ line. Output of the fxcor utility characterizes the crosscorrelation function, from which estimates of each star's radial velocity are easily inferred. Rotational velocities can also be estimated from the cross-correlation function full-width (CCF FWHM) using a procedure developed by Steinhauer (2003). This procedure exploits the relationship between the $\mathrm{CCF}$ FWHM, line widths and $V \sin i$, using a set of numerically "spun-up" standard spectra with comparable spectral types to constrain the relationship. For a significant fraction of our sample, higher rotational velocities conspired with weaker lines to produce spurious values of the radial velocity when the region near $\mathrm{H} \alpha$ was excluded; quoted values in our table include stars for which the cross-correlation included $\mathrm{H} \alpha$. For

\footnotetext{
6 IRAF is distributed by the National Optical Astronomy Observatory, which is operated by the Association of Universities for Research in Astronomy, Inc., under cooperative agreement with the National Science Foundation.
}

simplicity, $V_{\text {rot }}$ as used here implicitly includes the unknown $\sin i$ term.

There are two means to test the precision of the radial velocities, the size of the scatter among multiple observations of the same star and the scatter in residuals relative to an independent source of measurements. Over the multiple HYDRA runs, 36 stars were observed twice and two stars were observed three times. The dispersions in radial velocity for the latter pair are 0.70 and $0.55 \mathrm{~km} \mathrm{~s}^{-1}$. Among the stars with two observations, star 7112 showed a difference of $28 \mathrm{~km} \mathrm{~s}^{-1}$ between the two epochs and is clearly a double-lined spectroscopic binary. It sits among the giants at the level of the clump and undergoes eclipses (Arentoft et al. 2007). With both sets of lines visible, it is likely that the system is composed of two FRGs.

For the 35 remaining paired sets of observations, the average absolute difference in the radial velocities is $0.84 \pm 0.96 \mathrm{~km} \mathrm{~s}^{-1}$. However, the two pairs with the largest discrepancies, 3.22 and $4.62 \mathrm{~km} \mathrm{~s}^{-1}$, are stars 1359 , categorized by Mermilliod \& Mayor (2007) as a probable binary, and 4376, tagged as a probable binary in the current investigation due to the range in published velocities for this star. If these two are removed from the sample, the mean offset between paired observations becomes $0.65 \pm$ $0.57 \mathrm{~km} \mathrm{~s}^{-1}$.

We next compare our data with those of Mermilliod \& Mayor (2007), Mermilliod et al. (2008), which supersedes the smaller and/or less precise samples of Friel \& Janes (1993), Minniti (1995), Carretta et al. (2004), and Reddy et al. (2012), and with the more recent work of Carlberg (2014) and Carlberg et al. (2016). Of the 30 members and four non-members observed by Mermilliod \& Mayor (2007), all but two of the non-members and two of the members were included in our observations. For the remaining 30 stars, the mean residual in radial velocity, in the sense (MM-Table 1), is $+0.58 \pm 1.80 \mathrm{~km} \mathrm{~s}^{-1}$. If probable binaries 1359, 2251, and 4376 are removed, the mean residual drops to $+0.31 \pm 0.96 \mathrm{~km} \mathrm{~s}^{-1}$. It is encouraging to note that the 
dispersion is very comparable to that derived from a similar analysis of the giants and main sequence stars in NGC 6819 (LeeBrown et al. 2015). Our cluster mean velocity for the 25 members is $83.2 \pm 1.2 \mathrm{~km} \mathrm{~s}^{-1}$.

Carlberg (2014) has published radial velocities for 27 red giant members of NGC 2506; all but 2122 are included in the current study. From the residuals between the two samples, five stars stand out as anomalous, 2109 and 2276, as well as the already identified 1359, 2251, and 4376. If these stars are eliminated, the 21 remaining stars generate mean residuals of $+0.63 \pm 1.04$. Carlberg (2014) finds a mean radial velocity for these 21 giants of $+83.9 \pm 1.1 \mathrm{~km} \mathrm{~s}^{-1}$.

Finally, Carlberg et al. (2016) present observations of five red giants in NGC 2506, all of which were included in the three previous studies. While velocities of four of the stars are consistent with previous observations within the uncertainties, star 3265 exhibits a range from 80.3 to $85.3 \mathrm{~km} \mathrm{~s}^{-1}$. While not conclusive, the spread could be indicative of a binary classification, as noted by Carlberg et al. (2016), who also emphasize the peculiar location of this star in the CMD and the distinctly anomalous $[\mathrm{Fe} / \mathrm{H}]$ derived relative to the other four giants.

\section{Cluster Membership}

\subsection{Probable Binaries}

To minimize the potential distortions in any trends between main sequence and red giant stars, in addition to removing nonmembers, it is valuable to identify likely binaries which may have either anomalous colors and spectra, as well as modified evolution due to binary interaction. Among the giants, it has already been noted that stars 1359, 2109, 2251, 2276, 3265, and 4376 are probable binaries. Arentoft et al. (2007) used intermediate-band photometry to identify a large sample of variables in the field of NGC 2506, including three eclipsing binaries brighter than $V=15.6$. This sample includes the already noted giant, 7112, and two stars near the turnoff, 1136 and 1212, both of which appear to be radial-velocity cluster members based upon preliminary analysis of unpublished highresolution spectroscopy of these stars (Arentoft et al. 2007). Star 3255 (V10) is a red giant blueward of the clump with two almost identical radial-velocity measures, both consistent with cluster membership. Arentoft et al. (2007) find variability at the mmag level with a periodicity of 10 days $^{-1}$, though it does lie near the saturation limit of their photometric survey. As a member, it is clearly not a $\delta$ Scuti star.

A straightforward method for tagging potential binaries from single spectra is to look for a double set of lines within the spectrum, as revealed via fxcor or, at minimum, a consistent asymmetry in the line profile for a spectrum caused by the overlap of two lines. From visual and fxcor inspection of all the spectra, stars 1106, 2144, 2376, 3378, 4262, 5086, 7025, 7033, $7050,7057,7052,7101$, and both spectra for 7112 were noted as possible spectroscopic binaries.

It should be mentioned that the apparently low fraction of identified binaries $(<10 \%)$ is not indicative of the probable cluster fraction and is primarily tied to the combination of selection effects in the initial selection of probable cluster members at the turnoff, the limited number of radial-velocity measures per star, and the limits on the radial-velocity measurements for stars with higher than average rotation.

\subsection{Radial-velocity Membership}

With radial velocities for 287 stars but proper-motion membership for only 129, final membership for the majority of the sample is strongly dependent upon the single velocity component, weighted by location within the CMD. While this approach worked extremely well for NGC 6819 (Lee-Brown et al. 2015), the younger age by $0.4 \mathrm{Gyr}$ places the turnoff of NGC 2506 in a regime where rotational speeds can remain high (greater than $25 \mathrm{~km} \mathrm{~s}^{-1}$ ), significantly impacting the precision of the radial velocity. It should be noted that for the giants, the measured rotational velocity is dominated by the resolution of the spectra and therefore should be regarded as an upper limit to the true value. By contrast, Carlberg (2014) derived precision rotational velocities from $R \sim 44,000$ spectra from the MIKE spectrograph. From 21 single-star members common to our sample, Carlberg (2014) finds an average rotational speed of $3.2 \pm 1.2 \mathrm{~km} \mathrm{~s}^{-1}$; our lower $R$ data generate an average of $13.8 \pm 3.0 \mathrm{~km} \mathrm{~s}^{-1}$.

To determine membership by radial velocity alone, any star with a radial velocity more than three $\sigma$ from the cluster mean as defined by our single cluster giants $\left(83.3 \mathrm{~km} \mathrm{~s}^{-1}\right)$ will be classified as a probable non-member. To set the individual $\sigma$ for each star, two factors were taken into account. First, as expected, the calculated uncertainty in the radial velocity is well correlated in approximately linear fashion with the rotational speed. We averaged the calculated error in radial velocity as defined by fxcor and the error as predicted from $V_{\text {rot }}$, i.e., $\sigma_{V \text { rad }}=0.044^{*} V_{\text {rot }}+0.246$. Second, in the limit of perfect velocity measurements, this estimate implies no expected dispersion among the stars, ignoring the intrinsic velocity dispersion among the sample caused by motion about the center of mass of the cluster. Thus, the previously quoted dispersions among the purportedly single-star radial velocities for the red giants in our sample $\left(1.2 \mathrm{~km} \mathrm{~s}^{-1}\right)$ and that of Carlberg (2014) $\left(1.1 \mathrm{~km} \mathrm{~s}^{-1}\right)$ are combinations of both measurement errors and the intrinsic spread among the giants. The slight improvement in the dispersion for the data with higher resolution is not unexpected so, as an approximate estimate for the intrinsic radial velocity spread, we adopt $1.0 \mathrm{~km} \mathrm{~s}^{-1}$. This is effectively the same value we would get if we adopted $1.2 \mathrm{~km} \mathrm{~s}^{-1}$ for the total dispersion, but removed $0.6-0.7 \mathrm{~km} \mathrm{~s}^{-1}$ as the instrumental scatter derived from multiple observations of the same stars over different runs. The final adopted $\sigma$ in the individual $V_{\text {rad }}$ becomes the addition in quadrature of the fixed intrinsic cluster spread with the individual measurement error as derived above.

Application of the radial-velocity criterion to 265 probable non-binary stars leads to initial membership for 187 stars, though we emphasize that the claim to single-star status is based upon a lack of direct evidence for binarity, which is difficult to come by for stars with only one spectroscopic observation. Of these 187 stars, 98 have proper-motion probabilities, 86 of which are above $50 \%$. As the second cut, we eliminate the 12 stars with probability ranging from $0 \%$ to $42 \%$, leading to a final sample of 175 probable, single-star members. It is encouraging to note that of the 78 stars classified as radial-velocity non-members, 17 have proper-motion probabilities of which 13 lie below 50\%. The distribution of single-star members (solid black line) and non-members (dashed blue curve) based solely on radial velocity is shown in Figure 1. 


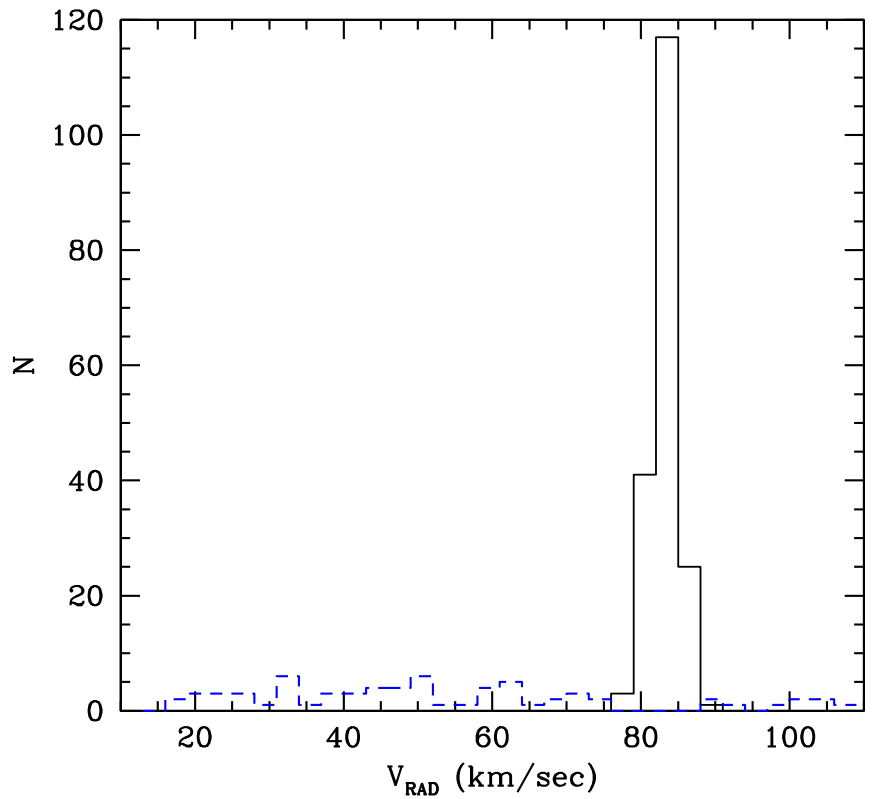

Figure 1. Distribution of radial velocities for single stars classified as probable radial-velocity members (solid black line) and potential non-members from radial velocity alone (blue dashed line).

\subsection{The CMD}

To optimize the precision of the photometric input for the atmospheric parameters needed for the spectroscopic analysis, particularly $T_{\text {eff }}$ from colors and $\log g$ from location within the CMD, use will be made of three photometric indices, $b-y$, $B-V$, and $h k$. For the color temperature, the $B-V$ values of Van Stockum et al. (2005, hereafter VS) defined the starting point. As a check on the photometric zero-point, comparison with the photoelectric observations of McClure et al. (1981) for 29 stars, 23 brighter than $V=16.0$, led to a mean offset of $-0.010 \pm 0.025$, in the sense (MC-VS), which was applied to the CCD $B-V$ system.

Photometric indices from the intermediate-band (AT16) and broad-band (VS) surveys are available for over 2000 stars. A cubic relation between $(b-y)$ and $(B-V)$ was determined using a set of 730 stars with the lowest color errors; the relationship has a standard deviation in $(B-V)$ color of 0.022 . A similar relationship was determined between $h k$ indices and $B-V$ using solely cool member giants (AT16) to provide an additional temperature measure.

The spectroscopic sample includes ten stars without Strömgren photometry due to placement slightly outside the field of the published Strömgren survey. For nine of these stars, the VS photometry was used exclusively. Conversely, the broad-band photometry suffered a few gaps, particularly at the very bright end. For four of the five stars lacking photometry from the VS survey, we relied exclusively on the Strömgren photometry. These few very bright stars targeted primarily the red giants, although one of the five bright stars, 1375, is clearly a hot star. For the one star lacking photometric indices from both surveys, 7007 (Tyc 5416-2526-1), Tycho values were used for $V$ and $B-V$.

From 273 nonvariable stars in the spectroscopic survey in common with VS, the mean difference in $V$ in the sense (AT16-VS) is $-0.009 \pm 0.021$. Since the AT16 $V$ system is in excellent agreement with that of McClure et al. (1981), where the mean difference in $V$ from 33 stars brighter than $V=17.0$

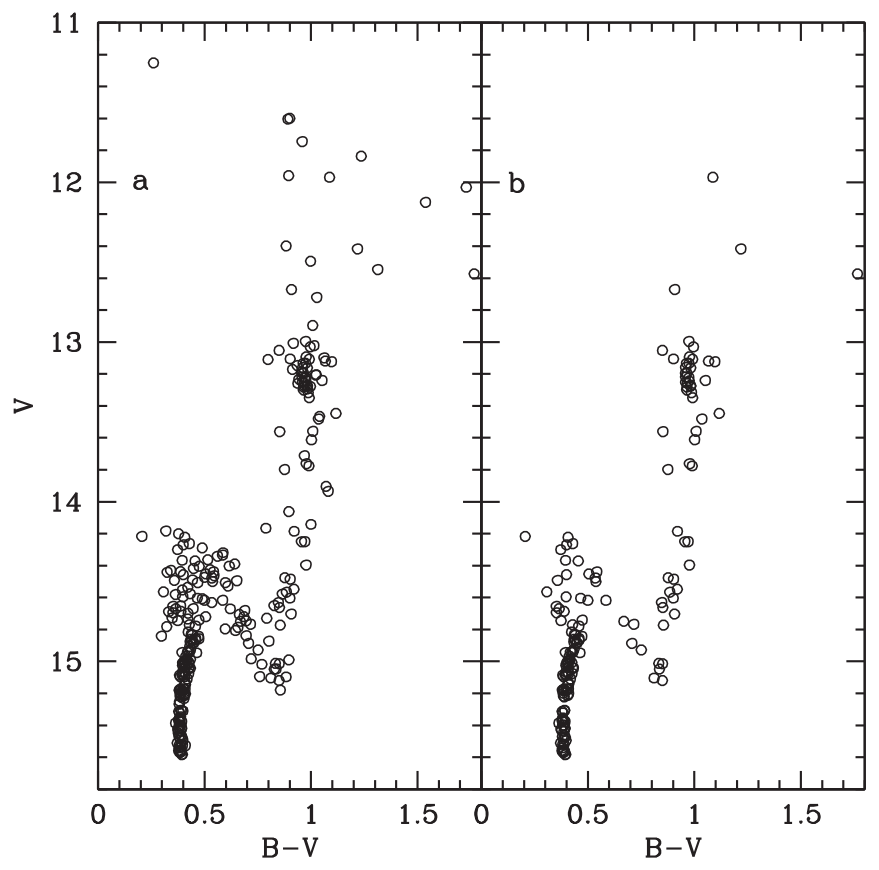

Figure 2. (a) CMD for all stars observed spectroscopically. (b) Probable singlestar members of the spectroscopic sample.

is $-0.001 \pm 0.034$ in the sense (MC-AT16), a simple average of AT16 and VS was adopted for $V$. For 9 of the 10 stars not surveyed by AT16, the $V$ magnitudes of VS were adopted without modification.

Figure 2(a) shows the CMD for all stars observed spectroscopically; Figure 2(b) is composed of NGC 2506 radialvelocity and, if available, proper-motion members for which no evidence of binarity currently exists. Table 1 summarizes the basic information about all stars observed spectroscopically. Stars listed as $\mathrm{M}$ are probable radial-velocity members, NM are non-members, $\mathrm{MB}$ are probable binary members, $\mathrm{MN}$ are radial-velocity members with proper-motion membership below $50 \%, \mathrm{~B}$ are probable binaries for which the radial velocity deviates significantly from the mean, and BNM and $\mathrm{BM}$ are binaries with deviant radial velocities but propermotion probabilities below and above $50 \%$, respectively.

What is apparent from a comparison of the two CMDs is that the greatest decline in stars due to the elimination of radialvelocity non-members is concentrated in two regions of the CMD. The first is near the top of the vertical turnoff, between $V=14.2$ and 14.8. A large sample from outside the original proper-motion survey region was selected from this region of the CMD in the hope of mapping the turnoff hook and the inital phase of evolution to the subgiant branch. The second concentration is among the red giants in the vertical band between $B-V=0.8$ and 1.2 , where stars were initially retained in the hope of adding to the cluster subgiant and red giant branches. While some of these stars, particularly brighter than the clump, proved to be non-members, 29 probable subgiant and giant members were added to the final sample. Again, both color regions will be contaminated by field stars at any age younger than $\sim 3$ Gyr for the turnoff and at almost any age for disk red giants and clump stars. Since we only have one velocity component for these stars, even with relatively tight radial-velocity limits, it is probable that a handful of field nonmembers are still contained within the member sample. 


\section{Metallicity}

Determination of stellar elemental abundances from high dispersion spectroscopy depends upon a variety of factors, from the signal-to-noise ratio $(\mathrm{S} / \mathrm{N})$ of the spectroscopic region under analysis to the fundamental stellar parameters defining the strength and appearance of the line profiles: $V_{\text {rot }}, T_{\text {eff }}, \log g$, and microturbulent velocity, $v_{t}$. With high resolution over an extended wavelength region and a large number of lines for both neutral and ionized elements, one can constrain the potential range of parameters by requiring a lack of trends between the derived abundances and the fundamental parameters, as well as the excitation potential. For a smaller spectral wavelength range with fewer lines, one is forced to fall back on alternative means of deriving the key parameters, independent of the spectrum, e.g., using a color-based $T_{\text {eff }}$ and $\log g$ defined by position in the CMD for cluster members.

Since the first cluster analysis in this series for NGC 3680 (Anthony-Twarog et al. 2009), where a modest number of mostly dwarf stars and a few giants were being considered, our approach has evolved to allow discussion of samples expanded by almost an order of magnitude compared to earlier work by us and others. A key change, as exemplified by NGC 6819 (Lee-Brown et al. 2015), has been the adoption of an automated line-measurement program, ROBOSPECT (Waters \& Hollek 2013), to replace exclusive dependence on manual measurement of line EWs as input in traditional model atmosphere analysis via MOOG (Sneden 1973). Because ROBOSPECT consistently performed as well as manual measurements over the color range from dwarfs to giants, the primary sources of uncertainty among the final abundances within a cluster ultimately depended upon the primary parameters of $T_{\text {eff }}$ and $v_{t}$. In theory, one could adjust these two scales for the stellar sample until the abundances showed no trend with either variable, but this would minimize the slope without supplying an independent check on the zero-point of the abundances. In particular, the color $-T_{\text {eff }}$ relations adopted to date for the dwarfs and giants come from two independent relations, each of which has its own slope and zero-point, leading to the possibility of a mismatch between these two subsamples within the same cluster. While one could check the derived parameters against those already published for cluster stars observed at high dispersion by others, in many cases the samples only discuss either dwarfs or giants, but not both, or little if any high dispersion work is available for the cluster of interest.

As an alternative to our photometric $T_{\text {eff }}$ values and EWbased spectroscopic $[\mathrm{Fe} / \mathrm{H}]$ estimates, we have attempted to derive $T_{\text {eff }}$ and $[\mathrm{Fe} / \mathrm{H}]$ for each star in our sample using ANNA (D. B. Lee-Brown 2018, in preparation), a new, flexible, Python-based code for automated stellar parameterization. ANNA utilizes a feed-forward, convolutional neural network (Arbib 2002), a machine-learning technique, to infer stellar parameters of interest from input spectra. Multiple tests show that ANNA is capable of producing accurate metallicity estimates with precision competitive with our EW-based analysis. Additionally, ANNA is capable of accurately inferring $T_{\text {eff }}$ from our spectra alone, providing an alternative temperature determination for each star. A deeper discussion of ANNA's design and capabilities will be given in D. B. LeeBrown (2018, in preparation), but we briefly summarize its operation here. ANNA is freely available for download; the version of ANNA used in this investigation can be found at
Zenodo (Lee-Brown 2017), while the current version of the code can be found at GitHub. ${ }^{7}$

\subsection{ANNA: A Neural Network for Temperatures and Abundances}

ANNA builds on previous studies examining the suitability of neural networks as tools to parameterize stellar spectra (e.g., Bailer-Jones et al. 1997; Allende Prieto et al. 2000; Snider et al. 2001; Manteiga et al. 2010; Dafonte et al. 2016; Li et al. 2017). The neural network used in ANNA consists of layers of sequential mathematical operations. The first, or input, layer is a stellar spectrum consisting of an ordered sequence of pixel values. These values are then summed according to many different sets of weight vectors, such that a set of weighted sums of the inputs is calculated. This collection of weighted sums forms a hidden layer. Each of the weighted sums in this hidden layer then serves as input to a nonlinear function. In ANNA, this function is the rectified linear unit (ReLU), defined as $f(x)=\max (x, 0)$, where $x$ denotes the weighted sum serving as input to the function. The outputs from this function are then used to construct another set of weighted summations, organized into another hidden layer, and the process repeats several times. The final result of this sequence of operations is a set of outputs $\left\{y_{i}\right\}$ which, in the case of ANNA, consists of the stellar parameters of interest that have been inferred from the input spectrum.

ANNA contains five layers: an input and an output layer connected via three sequential hidden layers. The first hidden layer is a convolutional layer which contains a collection of filters that are each convolved with the inputs. The filters consist of collections of weights used to construct a weighted sum that only depends on a localized subset of the layer inputs. The filters are windowed across the inputs to generate many such localized summations. This localization reduces the number of free parameters (weight values) in the first hidden layer, helping to reduce overfitting during training while improving computational performance (see Arbib 2002). The other two hidden layers are fully connected; each summation in these layers is connected via weights to all the ReLU operations in the previous layer.

ANNA's ability to translate between input spectra and accurate stellar parameters requires correct selection of each weight value used during the summation steps. This selection is done automatically through supervised learning. Initially, small weight values are sampled randomly from a normal distribution. The network is then provided an input consisting of a spectrum whose output parameters are known. ANNA will produce outputs based on the input spectrum; the accuracy of these reported outputs can be quantitatively assessed via a cost function, $J\left(y_{\text {true }}, y_{\text {infer }}\right)$, that measures the deviations of the reported outputs from the known stellar parameters. In ANNA, a quadratic cost function is used, $J=\Sigma\left(y_{\text {true }}-y_{\text {infer }}\right)^{2}$. Thus, for large deviations of the network outputs from the known values, the value of $J$ is correspondingly large. Minimization of $J$, therefore, implies that the networks weights are such that $y_{\text {infer }}$ closely matches $y_{\text {true }}$. This minimization is accomplished by computing the gradient of $J$ with respect to each network weight, followed by an update of the weights in the direction of lower $J$. Through successive iterations of this updating process, the weights eventually converge to optimal values. The

\footnotetext{
https://github.com/dleebrown/ANNA
} 
Table 2

ANNA Training Set Parameters

\begin{tabular}{ll}
\hline \hline Parameter & Range \\
\hline$T_{\text {eff }}(\mathrm{K})$ & $3500-7500$ \\
{$[\mathrm{Fe} / \mathrm{H}](\mathrm{dex})$} & -1.0 to 0.0 \\
$\log (g)(\mathrm{dex})$ & $2.0-4.5$ \\
$v_{t}\left(\mathrm{~km} \mathrm{~s}^{-1}\right)$ & $1.0-2.5$ \\
$V_{\text {rot }}\left(\mathrm{km} \mathrm{s}^{-1}\right)$ & $0-70$ \\
$R V\left(\mathrm{~km} \mathrm{~s}^{-1}\right)$ & -10 to 95 \\
$\mathrm{~S} / \mathrm{N}$ & $100-250$ \\
\hline
\end{tabular}

network will then be capable of inferring stellar parameters from spectra whose parameterizations are unknown.

We trained ANNA using continuum-normalized spectra generated with the code SPECTRUM (Gray \& Corbally 1994), Kurucz atmospheric models (Kurucz 1992), and a custom linelist generated using VALD (Kupka et al. 1999) that was adjusted to reproduce the solar spectrum. We generated 15,000 high-resolution $(R \sim 670,000)$ training spectra with parameters randomly selected between the ranges given in Table 2 . The high-resolution spectra were then post-processed to better mimic our sample of HYDRA spectra. This post-processing included random radial-velocity shifts, rotational broadening, and smoothing using a Gaussian line-spread function to a resolution of $R \sim 9000$. This resolution is lower than the actual resolution of our spectra ( $R \sim 13,000)$, and was selected during testing of ANNA as it represents the resolution corresponding to the minimum average rms deviation between our synthetic training spectra and a sample of real Hydra spectra. This adoption of a lower-than-actual resolution for our training spectra likely indicates that our synthetic models imperfectly reproduce the line-spread function of the spectrograph and/or do not completely model the subtle broadening effects due to changes in surface gravity or microturbulent velocity. However, during testing we ultimately determined that ANNA's temperature and metallicity determinations were relatively insensitive to the adopted training resolution; our ANNA results are materially unchanged if we were to instead adopt $R \sim 13,000$ during training. This is likely due to the fact that temperature and metallicity are more sensitive to relative line strengths, rather than the particular shapes of the line profiles.

The pixel scale was set to $0.2 \AA \mathrm{px}^{-1}$. We also limited the wavelength coverage of our training spectra to $6625-6825 \AA$. This was done to avoid having to model calibration artifacts in our real spectra in the region of $\mathrm{H} \alpha$. After post-processing, our training sample consisted of 225,000 spectra.

During training, subsamples of 100 randomly selected example spectra were used during each weight update iteration. When spectra were selected for training, a wavelengthdependent amount of noise was added according to a randomly selected $\mathrm{S} / \mathrm{N}$ value and a relative $\mathrm{S} / \mathrm{N}$ template derived from HYDRA observations of the Sun. Additionally, small, random continuum offsets were added to simulate continuum placement errors in our real spectra. Training was carried out until the network cost function failed to improve within 20,000 weight update iterations, for a total of approximately 200,000 iterations.

After training, we verified the capabilities of the trained network using a sample of real HYDRA spectra. This sample included spectra of the Sun, as well as members of the open clusters NGC 6819 (Lee-Brown et al. 2015) and the Hyades
(Cummings et al. 2017). These test spectra were first linearly interpolated onto the wavelength grid used during the training process. Of the potential stellar parameters, the trained network most reliably determined the correct $T_{\text {eff }}$ and $[\mathrm{Fe} / \mathrm{H}]$; this is unsurprising as these two parameters contribute most strongly to the observable features in our selected spectral range. Hotter stars in our test sample $\left(T_{\text {eff }} \sim 6500 \mathrm{~K}\right)$ could not be accurately parameterized by ANNA, likely due to a lack of strong spectral features over the wavelength range of interest. For cooler stars, we verified that ANNA returned reliable parameter determinations down to $T_{\text {eff }} \sim 4000 \mathrm{~K}$ using NGC 6819 spectra. With the exception of one star (4402), which we omit from our ANNA analysis, the stars in our NGC 2506 sample have surface temperatures well above this tested value.

ANNA's $T_{\text {eff }}$ and $[\mathrm{Fe} / \mathrm{H}]$ results derived from the cooler $\left(T_{\text {eff }} \leqslant 6500 \mathrm{~K}\right)$ test spectra compare favorably with parameters known a priori. For a sample of 89 solar spectra of typical $\mathrm{S} / \mathrm{N}=200$ obtained from the daytime sky with HYDRA for calibration purposes during our observing runs for NGC 2506, ANNA returns an average $T_{\text {eff }}=5780 \pm 77 \mathrm{~K}$ (s.d.) and average $[\mathrm{Fe} / \mathrm{H}]=0.04 \pm 0.03$ dex (s.d.). From the rich sample of 37 giants and 184 dwarfs in NGC 6819, ANNA derives $[\mathrm{Fe} / \mathrm{H}]=-0.01 \pm 0.09$ (s.d.) and $[\mathrm{Fe} / \mathrm{H}]=-0.06 \pm 0.08$ (s.d.), respectively, in excellent agreement with the EW analysis (Lee-Brown et al. 2015) which generates $[\mathrm{Fe} / \mathrm{H}]=$ $-0.03 \pm 0.09$ (s.d.). Finally, despite the Hyades' super-solar metallicity, $[\mathrm{Fe} / \mathrm{H}] \sim 0.15$ (see the discussion in Cummings et al. 2017), which places it outside the range of metallicities used during training, ANNA correctly infers $[\mathrm{Fe} / \mathrm{H}]=$ $0.17 \pm 0.05$ (s.d.). These results collectively indicate ANNA's reliability over a range in stellar $T_{\text {eff }}$ and $[\mathrm{Fe} / \mathrm{H}]$ typical of disk clusters.

\subsection{ANNA Parameters: NGC 2506}

The initial database of spectra processed through ANNA consisted of the 175 dwarfs and giants classed as single-star members, as detailed in Table 1. It quickly became apparent that the stars at the turnoff, even with $T_{\text {eff }}$ below $6500 \mathrm{~K}$, generated results which were inconsistent and/or subject to larger than desirable errors. Since the same conclusion was reached after using ROBOSPECT, independent of ANNA, and by tests using manual EW measurements for a representative subset of turnoff stars, the source of the problem lies with the spectra rather than the technique adopted. In contrast with our work on NGC 6819, the fundamental weaknesses of the dwarf analyses in NGC 2506 are due to the lower metallicity of the cluster, the hotter $T_{\text {eff }}$ due to the younger age combined with a lower metallicity, and the significantly wider range of $V_{\text {rot }}$ In fact, the large majority of stars at the turnoff of NGC 6819 have $V_{\text {rot }}$ below $25 \mathrm{~km} \mathrm{~s}^{-1}$ (C. P. Deliyannis et al. 2018, in preparation), in contrast with NGC 2506 where this value defines an approximate lower bound for the majority of stars at the turnoff.

We therefore used ANNA to derive $T_{\text {eff }}$ and $[\mathrm{Fe} / \mathrm{H}]$ only for stars with $(B-V)_{0}$ between 0.65 and 1.15 , excluding only one cool giant with $(B-V)_{0}$ greater than 1.7. The results for 62 red giant members are presented in Table 3 under the columns $T_{\mathrm{NN}}$ and $[\mathrm{Fe} / \mathrm{H}]_{\mathrm{NN}}$. From these 62 stars, we first construct a mean $[\mathrm{Fe} / \mathrm{H}]$ value $=-0.25 \pm 0.13$ (s.d.). The majority of the scatter is caused by three stars which have abundances ranging from solar to twice solar. These metallicities are consistent with those derived from ROBOSPECT measures and traditional EW 
Table 3

Abundances for NGC 2506 Stars

\begin{tabular}{|c|c|c|c|c|c|c|c|c|c|c|c|c|}
\hline ID No. & $T_{\mathrm{NN}}$ & {$[\mathrm{Fe} / \mathrm{H}]_{\mathrm{NN}}$} & {$[\mathrm{Fe} / \mathrm{H}]_{\mathrm{RS}}$} & MAD & $N_{\text {lines }}$ & {$[\mathrm{Ca} / \mathrm{H}]$} & {$[\mathrm{Si} / \mathrm{H}]$} & {$[\mathrm{Ni} / \mathrm{H}]$} & $\sigma([\mathrm{Ni} / \mathrm{H}])$ & $T_{\text {phot }}$ & $\log g$ & $\overline{v_{t}}$ \\
\hline 1108 & 5448 & -0.21 & -0.13 & 0.14 & 16 & 0.00 & -0.34 & -0.32 & 0.11 & 5372 & 3.23 & 1.35 \\
\hline 1112 & 4995 & -0.26 & -0.26 & 0.06 & 16 & $\ldots$ & -0.28 & -0.34 & 0.04 & 5070 & 2.24 & 1.69 \\
\hline 1229 & 4821 & -0.34 & -0.34 & 0.06 & 16 & $\ldots$ & -0.39 & -0.42 & 0.08 & 4856 & 2.32 & 1.65 \\
\hline 1301 & 5358 & -0.29 & -0.34 & 0.09 & 16 & -0.19 & -0.40 & -0.36 & 0.05 & 5249 & 3.23 & 1.35 \\
\hline 1320 & 4971 & -0.26 & -0.25 & 0.04 & 16 & $\ldots$ & -0.29 & -0.39 & 0.03 & 5051 & 2.43 & 1.62 \\
\hline 1325 & 4967 & -0.30 & -0.29 & 0.07 & 16 & $\ldots$ & -0.34 & -0.42 & 0.02 & 5070 & 2.34 & 1.65 \\
\hline 1340 & 5090 & -0.33 & -0.24 & 0.09 & 16 & 0.07 & -0.28 & -0.29 & 0.04 & 5308 & 3.18 & 1.37 \\
\hline 1377 & 5489 & -0.24 & -0.24 & 0.12 & 16 & -0.01 & -0.39 & -0.29 & 0.07 & 5421 & 3.29 & 1.36 \\
\hline 2212 & 4824 & -0.27 & -0.31 & 0.04 & 15 & $\ldots$ & -0.37 & -0.46 & 0.03 & 4810 & 1.90 & 1.60 \\
\hline 2255 & 4933 & -0.31 & -0.26 & 0.06 & 16 & 0.05 & -0.37 & -0.42 & 0.06 & 5063 & 2.72 & 1.51 \\
\hline 2309 & 5061 & -0.25 & -0.29 & 0.04 & 16 & $\ldots$ & -0.32 & -0.40 & 0.04 & 5065 & 2.31 & 1.66 \\
\hline 2329 & 5092 & -0.26 & -0.28 & 0.06 & 16 & 0.11 & -0.36 & -0.38 & 0.01 & 5106 & 2.35 & 1.65 \\
\hline 2351 & $\ldots$ & $\ldots$ & 0.14 & 0.29 & 10 & -0.06 & -0.07 & -0.02 & 0.10 & 7079 & 3.67 & 2.30 \\
\hline 2364 & 5289 & -0.24 & -0.14 & 0.07 & 16 & 0.28 & -0.26 & -0.27 & 0.02 & 5377 & 2.28 & 1.73 \\
\hline 2375 & 5058 & -0.16 & -0.30 & 0.07 & 16 & $\ldots$ & -0.25 & -0.41 & 0.02 & 4990 & 2.60 & 1.55 \\
\hline 2380 & 5031 & -0.23 & -0.22 & 0.05 & 16 & $\ldots$ & -0.37 & -0.36 & 0.06 & 5094 & 2.33 & 1.66 \\
\hline 2401 & 5015 & -0.26 & -0.25 & 0.08 & 16 & $\ldots$ & -0.28 & -0.38 & 0.07 & 5111 & 2.37 & 1.65 \\
\hline 2402 & 4546 & -0.34 & -0.41 & 0.06 & 16 & $\ldots$ & -0.27 & -0.50 & 0.05 & 4504 & 1.90 & 1.60 \\
\hline 3110 & 5383 & -0.25 & -0.24 & 0.15 & 15 & 0.02 & -0.34 & -0.31 & 0.05 & 5364 & 3.23 & 1.35 \\
\hline 3204 & 5235 & -0.29 & -0.21 & 0.08 & 16 & 0.04 & -0.34 & -0.35 & 0.07 & 5231 & 2.04 & 1.60 \\
\hline 3231 & 5022 & -0.28 & -0.27 & 0.04 & 16 & 0.04 & -0.30 & -0.37 & 0.04 & 5096 & 2.33 & 1.66 \\
\hline 3243 & 5467 & -0.21 & -0.15 & 0.09 & 16 & 0.05 & -0.41 & -0.29 & 0.04 & 5437 & 3.23 & 1.35 \\
\hline 3324 & 5045 & -0.24 & -0.24 & 0.05 & 16 & $\ldots$ & -0.27 & -0.36 & 0.03 & 5089 & 2.43 & 1.62 \\
\hline 3356 & 5249 & -0.25 & -0.27 & 0.06 & 16 & 0.05 & -0.42 & -0.34 & 0.02 & 5200 & 2.99 & 1.42 \\
\hline 3359 & 5067 & -0.27 & -0.23 & 0.06 & 16 & $\ldots$ & -0.24 & -0.38 & 0.04 & 5106 & 2.41 & 1.63 \\
\hline 3392 & 5096 & -0.27 & -0.27 & 0.07 & 16 & 0.06 & -0.35 & -0.37 & 0.05 & 5166 & 2.32 & 1.69 \\
\hline 4109 & 5364 & -0.27 & -0.24 & 0.07 & 16 & 0.07 & -0.30 & -0.41 & 0.06 & 5311 & 2.75 & 1.54 \\
\hline 4128 & 5255 & -0.27 & -0.26 & 0.08 & 17 & 0.05 & -0.39 & -0.48 & 0.07 & 5246 & 2.32 & 1.69 \\
\hline 4129 & $\ldots$ & $\ldots$ & -0.28 & 0.06 & 5 & -0.45 & -0.11 & -0.25 & 0.18 & 6314 & 3.54 & 1.83 \\
\hline 4138 & 5030 & -0.29 & -0.27 & 0.06 & 16 & $\ldots$ & -0.27 & -0.34 & 0.03 & 5094 & 2.43 & 1.62 \\
\hline 4143 & 5058 & -0.22 & -0.31 & 0.08 & 16 & 0.05 & -0.34 & -0.43 & 0.03 & 5077 & 2.43 & 1.62 \\
\hline 4205 & 5082 & -0.24 & -0.21 & 0.09 & 16 & $\ldots$ & -0.29 & -0.34 & 0.04 & 5094 & 2.42 & 1.63 \\
\hline 4223 & $\ldots$ & $\ldots$ & -0.30 & 0.06 & 12 & -0.18 & -0.28 & -0.43 & 0.13 & 5886 & 3.42 & 1.48 \\
\hline 4240 & 4992 & -0.28 & -0.29 & 0.07 & 15 & 0.03 & -0.35 & -0.43 & 0.02 & 5030 & 2.43 & 1.62 \\
\hline 4274 & 5168 & -0.26 & -0.27 & 0.08 & 16 & $\ldots$ & -0.16 & -0.36 & 0.04 & 5035 & 2.73 & 1.50 \\
\hline 4372 & $\ldots$ & $\ldots$ & -0.40 & 0.18 & 6 & -0.54 & -1.14 & -0.85 & 0.18 & 6607 & 3.66 & 2.06 \\
\hline 4528 & 5534 & -0.19 & -0.22 & 0.09 & 16 & -0.05 & -0.33 & -0.38 & 0.06 & 5374 & 3.23 & 1.37 \\
\hline 5249 & 5526 & -0.34 & -0.55 & 0.17 & 16 & -0.50 & -0.50 & -0.65 & 0.09 & 5413 & 3.23 & 1.35 \\
\hline 5271 & 5059 & -0.26 & -0.25 & 0.04 & 16 & 0.10 & -0.36 & -0.38 & 0.02 & 5108 & 2.38 & 1.64 \\
\hline 5371 & 5401 & -0.23 & -0.18 & 0.07 & 16 & -0.05 & -0.46 & -0.30 & 0.04 & 5385 & 3.27 & 1.36 \\
\hline 7008 & 5423 & -0.28 & -0.34 & 0.10 & 16 & -0.05 & -0.33 & -0.33 & 0.09 & 5234 & 3.31 & 1.31 \\
\hline 7019 & 5234 & -0.26 & -0.21 & 0.06 & 16 & -0.06 & -0.39 & -0.35 & 0.09 & 5202 & 3.20 & 1.35 \\
\hline 7023 & 5090 & -0.25 & -0.29 & 0.06 & 16 & 0.04 & -0.35 & -0.47 & 0.04 & 5068 & 2.40 & 1.63 \\
\hline 7026 & 5344 & -0.23 & -0.34 & 0.06 & 16 & -0.17 & -0.31 & -0.46 & 0.06 & 5065 & 3.12 & 1.36 \\
\hline 7031 & 5611 & -0.48 & -0.43 & 0.17 & 11 & -0.34 & -0.34 & -0.48 & 0.07 & 5744 & 3.42 & 1.41 \\
\hline 7032 & $\ldots$ & $\ldots$ & -0.18 & 0.22 & 10 & -0.19 & -0.13 & -0.30 & 0.19 & 6161 & 3.65 & 1.64 \\
\hline 7036 & 5009 & -0.26 & -0.23 & 0.07 & 16 & -0.10 & -0.36 & -0.41 & 0.07 & 5051 & 2.42 & 1.62 \\
\hline 7041 & 5768 & 0.31 & 0.24 & 0.08 & 16 & $\ldots$ & 0.28 & 0.31 & 0.06 & 5643 & 3.58 & 1.31 \\
\hline 7043 & 5283 & -0.34 & -0.27 & 0.07 & 15 & 0.02 & -0.41 & -0.22 & 0.03 & 5377 & 3.14 & 1.40 \\
\hline 7045 & 4736 & -0.13 & -0.31 & 0.04 & 15 & $\ldots$ & -0.04 & -0.28 & 0.02 & 4741 & 2.52 & 1.57 \\
\hline 7048 & 4981 & -0.28 & -0.32 & 0.07 & 16 & $\ldots$ & -0.30 & -0.43 & 0.03 & 5021 & 2.27 & 1.67 \\
\hline 7066 & $\ldots$ & $\ldots$ & -0.05 & 0.20 & 13 & -0.54 & -0.38 & -0.21 & 0.09 & 6761 & 3.70 & 2.30 \\
\hline 7069 & 5478 & 0.34 & 0.24 & 0.01 & 15 & $\ldots$ & 0.27 & 0.08 & 0.09 & 5369 & 2.60 & 1.60 \\
\hline 7080 & 5377 & -0.24 & -0.21 & 0.10 & 15 & -0.05 & -0.32 & -0.38 & 0.10 & 5288 & 3.23 & 1.35 \\
\hline 7082 & 5712 & -0.31 & -0.27 & 0.16 & 13 & -0.22 & -0.31 & -0.38 & 0.19 & 5771 & 3.42 & 1.42 \\
\hline 7084 & 4750 & -0.29 & -0.34 & 0.04 & 15 & $\ldots$ & -0.41 & -0.48 & 0.02 & 4787 & 2.33 & 1.64 \\
\hline 7085 & 4959 & -0.29 & -0.30 & 0.06 & 16 & $\ldots$ & -0.21 & -0.43 & 0.04 & 4928 & 2.55 & 1.56 \\
\hline 7086 & 5247 & -0.37 & -0.17 & 0.11 & 16 & -0.13 & -0.23 & -0.28 & 0.10 & 5479 & 3.40 & 1.33 \\
\hline 7088 & $\ldots$ & $\ldots$ & -0.31 & 0.19 & 6 & -0.31 & -0.63 & -0.43 & 0.18 & 6329 & 3.54 & 1.83 \\
\hline 7093 & 4989 & 0.04 & 0.02 & 0.11 & 16 & $\ldots$ & -0.02 & -0.15 & 0.07 & 5244 & 3.17 & 1.36 \\
\hline 7098 & 5099 & -0.30 & -0.34 & 0.04 & 15 & 0.01 & -0.29 & -0.49 & 0.05 & 5030 & 2.45 & 1.61 \\
\hline 7099 & 5055 & -0.25 & -0.29 & 0.05 & 16 & 0.04 & -0.31 & -0.43 & 0.06 & 5042 & 2.45 & 1.61 \\
\hline 7102 & 5154 & -0.22 & -0.27 & 0.06 & 16 & $\ldots$ & -0.35 & -0.38 & 0.02 & 5070 & 2.39 & 1.63 \\
\hline 7106 & 4848 & -0.28 & -0.33 & 0.06 & 15 & $\ldots$ & -0.35 & -0.50 & 0.06 & 4891 & 2.40 & 1.62 \\
\hline
\end{tabular}


Table 3

(Continued)

\begin{tabular}{lcccccccccccc}
\hline \hline ID No. & $T_{\mathrm{NN}}$ & {$[\mathrm{Fe} / \mathrm{H}]_{\mathrm{NN}}$} & {$[\mathrm{Fe} / \mathrm{H}]_{\mathrm{RS}}$} & $\mathrm{MAD}$ & $N_{\text {lines }}$ & {$[\mathrm{Ca} / \mathrm{H}]$} & {$[\mathrm{Si} / \mathrm{H}]$} & {$[\mathrm{Ni} / \mathrm{H}]$} & $\sigma([\mathrm{Ni} / \mathrm{H}])$ & $T_{\text {phot }}$ & $\log g$ & $v_{t}$ \\
\hline 7108 & 4959 & -0.29 & -0.32 & 0.07 & 16 & $\ldots$ & -0.30 & -0.41 & 0.01 & 5007 & 2.63 & 1.54 \\
7114 & 5001 & -0.33 & -0.31 & 0.06 & 16 & -0.07 & -0.41 & -0.32 & 0.09 & 5080 & 3.03 & 1.39 \\
7117 & 5085 & -0.24 & -0.27 & 0.05 & 16 & $\ldots$ & -0.33 & -0.38 & 0.04 & 5051 & 2.42 & 1.62 \\
7128 & 5228 & -0.25 & -0.24 & 0.07 & 15 & $\ldots$ & -0.32 & -0.34 & 0.02 & 5115 & 3.03 & 1.40 \\
\hline
\end{tabular}

(This table is available in machine-readable form.)

analyses. The implication is that these are, in fact, likely field stars with distinctly higher $[\mathrm{Fe} / \mathrm{H}]$ than the cluster. If these three stars are dropped, the revised cluster metallicity average and standard deviation from ANNA analysis becomes $[\mathrm{Fe} / \mathrm{H}]=-0.27 \pm 0.06$ (s.d.). The median of individual stellar $[\mathrm{Fe} / \mathrm{H}]$ values is robustly estimated as $-0.26 \pm 0.03$ with an error estimate based on a median of star-by-star absolute deviations from the cluster $[\mathrm{Fe} / \mathrm{H}]$ value.

\subsection{Robospect}

In keeping with our approach to spectroscopic abundance determination for previous clusters in this program, our default scheme for determining model atmosphere input temperatures was based upon photometric color, specifically $B-V$, defined in the current investigation as the average of the observed $B-V, b-y$ converted to $B-V$, and, when available for cluster members, $h k$ converted to $B-V$ for red giant members of the cluster. As noted earlier, the $T_{\text {eff }}$ for each star had been based on two primary color-temperature calibrations.

For dwarfs, the adopted calibration is that of Deliyannis et al. (2002), consistent with previous and ongoing spectroscopic studies by this group and compared in detail with more recent $T_{\text {eff }}$ calibrations in Cummings et al. (2017), namely:

$$
\begin{aligned}
T_{\text {eff }}(K)= & 8575-5222.7(B-V)_{0}+1380.92(B-V)_{0}^{2} \\
& +701.7(B-V)_{0}[[\mathrm{Fe} / \mathrm{H}]-0.15] .
\end{aligned}
$$

In previous investigations, the giant star color-temperature calibration of Ramírez and Meléndez (2005) was used for stars with $B-V>0.5$. With the availability of the revised $T_{\text {eff }}$ estimates produced by ANNA for subgiants and giants, we have replaced the cool-star calibration with the newer values. To mesh the scales smoothly, the reddening-corrected $(B-V)_{0}$ values for dwarfs have been run through the dwarf $T_{\text {eff }}$ calibration for all stars bluer than $(B-V)_{0}=0.34$, adopting $[\mathrm{Fe} / \mathrm{H}]=-0.30$. For stars redder than $(B-V)_{0}=$ 0.7 to $(B-V)_{0}=1.2$, we have adopted the ANNA $T_{\text {eff }}$ values, excluding the three stars which appear to be metal-rich from both ANNA and ROBOSPECT analyses. These data were then fit with a cubic relation:

$$
\begin{aligned}
T_{\text {eff }}(K)= & 8751.5-6955.7(B-V)_{0} \\
& +4519.1(B-V)_{0}^{2}-1461.1(B-V)_{0}^{3} .
\end{aligned}
$$

Since the relation is tied to a specific $[\mathrm{Fe} / \mathrm{H}]$, it is appropriate for NGC 2506 alone.

From the 107 single-star dwarfs, the mean $T_{\text {eff }}$ offset, in the sense (OLD-NEW), is $-0.2 \pm 6.5 \mathrm{~K}$. For 53 giants, the analogous comparison between ANNA $T_{\text {eff }}$ and the mean relation above is $+0.3 \pm 92.5 \mathrm{~K}$. If all giants, including the three anomalous stars, are compared, the values become $+0.7 \pm 98.9 \mathrm{~K}$.

Surface gravity estimates (log $g$ ) were obtained by direct comparison of $V$ magnitudes and $B-V$ colors to isochrones of VandenBerg et al. (2006), constructed for a scaled solar composition with $[\mathrm{Fe} / \mathrm{H}]=-0.29$ and an age of $1.85 \mathrm{Gyr}$, the same as the comparison presented in AT16. The isochrone's predicted magnitudes and colors were adjusted to match the cluster's reddening, $E(B-V)=0.058$ and apparent distance modulus, 12.75 (AT16).

Input estimates for the microturbulent velocity parameter were constructed using three prescriptions. For dwarfs within appropriate limits of $T_{\text {eff }}$ and $\log g$, the formula of Edvardsson et al. (1993) was used. For giants with $\log g<3.0$, a gravitydependent formula, $v_{t}=2.0-0.2 \log g$, was used. For subgiants and fainter red giants, we made use of a scheme developed by Bruntt et al. (2012) to analyze spectra of Kepler candidate G and $\mathrm{K}$ stars for which $T_{\text {eff }}$ from colors and $\log g$ validated by asteroseismology were available. The formulation by Bruntt et al. (2012) produces $v_{t}$ estimations from a $T_{\text {eff }}$ and $\log g$ dependent formulation that meshes well with the Edvardsson et al. (1993) and our previously used formula for giants if incremented by $0.2 \mathrm{~km} \mathrm{~s}^{-1}$.

A detailed discussion and application of the ROBOSPECT software (Waters \& Hollek 2013) and its optimization as applied to more than 330 stars in NGC 6819 are supplied in Lee-Brown et al. (2015). In short, our procedure consists of identification of the measurable spectroscopic absorption lines and calibration of atomic data, automated EW measurement using ROBOSPECT (Waters \& Hollek 2013), atmospheric model construction using parameters $\left(T_{\text {eff }}, \log g\right.$, and $\left.v_{t}\right)$ as described above, and chemical abundance analysis using MOOG (Sneden 1973).

Our ROBOSPECT line list is the same as used in the analysis of NGC 6819 (Lee-Brown et al. 2015) and contains 22 lines: $17 \mathrm{Fe} \mathrm{I}, 3 \mathrm{NiI}, 1 \mathrm{SiI}$, and $1 \mathrm{CaI}$. These lines are unblended in the solar spectrum and have solar EW values in the range 10-150 $\mathrm{mA}$, making them ideal for EW-based abundance analysis. For each line, we adopted atomic data (wavelength, excitation potential, and $\log g f$ ) contained in the VALD database (Kupka et al. 1999). We then modified the retrieved $\log g f$ values such that our solar EW measures reproduced the solar elemental abundances given in the 2010 version of MOOG, using a Kurucz (1992) model with $5770 \mathrm{~K}$, $4.40,1.14 \mathrm{~km} \mathrm{~s}^{-1}$, and 0.00 for $T_{\text {eff }}, \log g, v_{t}$, and $[\mathrm{Fe} / \mathrm{H}]$, respectively.

Using our calibrated line list, ROBOSPECT then iteratively determines the continuum, noise, and line components of a spectrum and reliably returns EWs that compare favorably with manual measurement of the lines. ROBOSPECT fits Gaussian line profiles, so we restrict our EW analysis to stars with projected rotation velocities $V_{\text {rot }} \leqslant 30 \mathrm{~km} \mathrm{~s}^{-1}$ in order to 


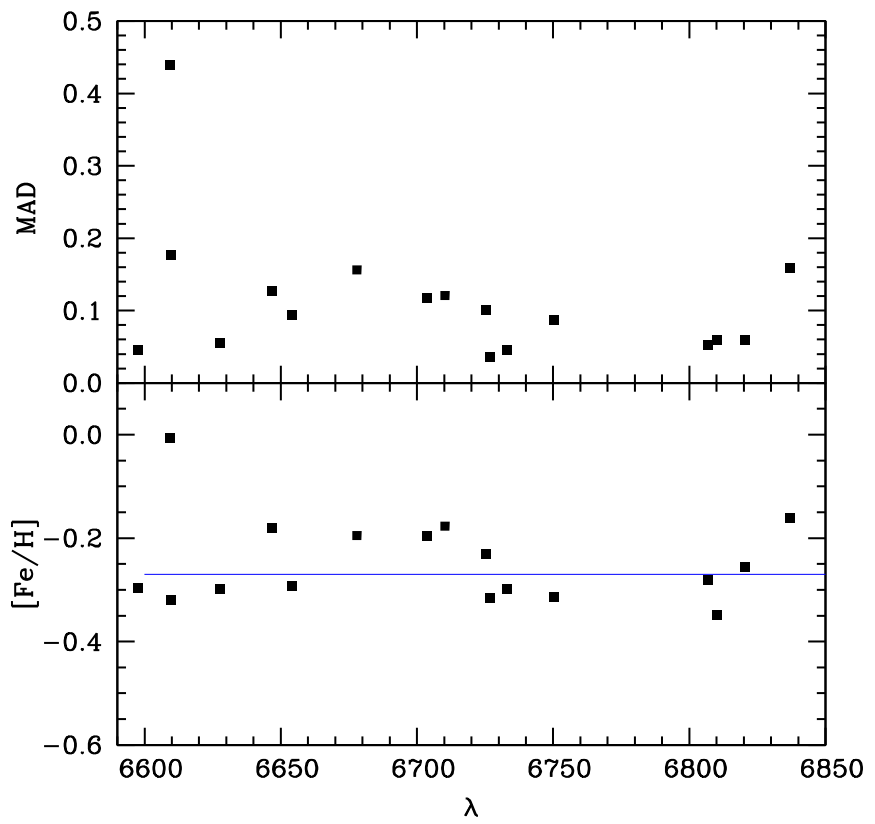

Figure 3. For each of the $17 \mathrm{Fe}$ lines the abundance $[\mathrm{Fe} / \mathrm{H}]$ is shown as well as the MAD (median absolute deviation) for our spectroscopic sample. The blue horizontal line shows the cluster sample median value of $[\mathrm{Fe} / \mathrm{H}]=n-0.27$.

reduce systematic EW offsets due to non-Gaussian profiles. Additionally, we reject EWs greater than $150 \mathrm{~m} \AA$ to restrict our analysis to lines corresponding to the linear portion of the curve of growth. Finally, we discard EWs that are within $3 \sigma$ of the local noise level (calculated using a $6 \AA$ window) to prevent introduction of spurious EW measurements into our analysis.

Our sample of stars processed with ROBOSPECT began with 78 spectra drawn from the sample summarized in Table 1, choosing stars with $\mathrm{M}$ or $\mathrm{MN}$ designations and rotational velocities $\leqslant 30 \mathrm{~km} \mathrm{~s}^{-1}$. Application of EW quality cuts left EW measures for $1022 \mathrm{Fe}, 207 \mathrm{Ni}, 52 \mathrm{Ca}$, and $70 \mathrm{Si}$ lines out of a possible $1326,234,78$, and 78, respectively. The majority of rejected $\mathrm{Fe}$ and $\mathrm{Ni}$ lines fell within our $3 \sigma$ significance threshold, while rejected $\mathrm{Ca}$ measurements generally had EWs $>150 \mathrm{m \AA}$. Our EW $<150 \mathrm{m \AA}$ criterion is more stringent than the EW $<200 \mathrm{m \AA}$ threshold used in Lee-Brown et al. (2015), but adopting the more generous threshold does not change our abundance results. Nine of the 78 stars had fewer than five Fe lines remaining for analysis and were not included in the cluster average, as was one additional star with a $B-V=1.77, T_{\text {eff }}$ below $4000 \mathrm{~K}$, and a spectrum dominated by molecular bands. Atmospheric parameters and EW abundances for each of the remaining 68 stars are provided in Table 3.

To translate between measured $\mathrm{EW}$ and $[\mathrm{A} / \mathrm{H}]$, we first constructed a 1D, plane-parallel model atmosphere for each star in our final ROBOSPECT sample using the Kurucz (1992) model grid and the $T_{\text {eff }}, \log g$, and $v_{t}$ values derived from our photometric observations. One star, 5270, had a temperature ( $7837 \mathrm{~K})$ greater than supported by the model atmosphere grid and was omitted from our sample. Our atmospheric models were then used in conjunction with our EW measurements as inputs to the abfind MOOG routine, resulting in an $[\mathrm{A} / \mathrm{H}]$ estimate for each of our measured EWs. As shown in Figures 3 and 4 , respectively, there are no apparent trends of $[\mathrm{Fe} / \mathrm{H}]$ with $T_{\text {eff }}$ or wavelength. There is, however, a serious decline in the number of turnoff stars with measurable abundances. The entire

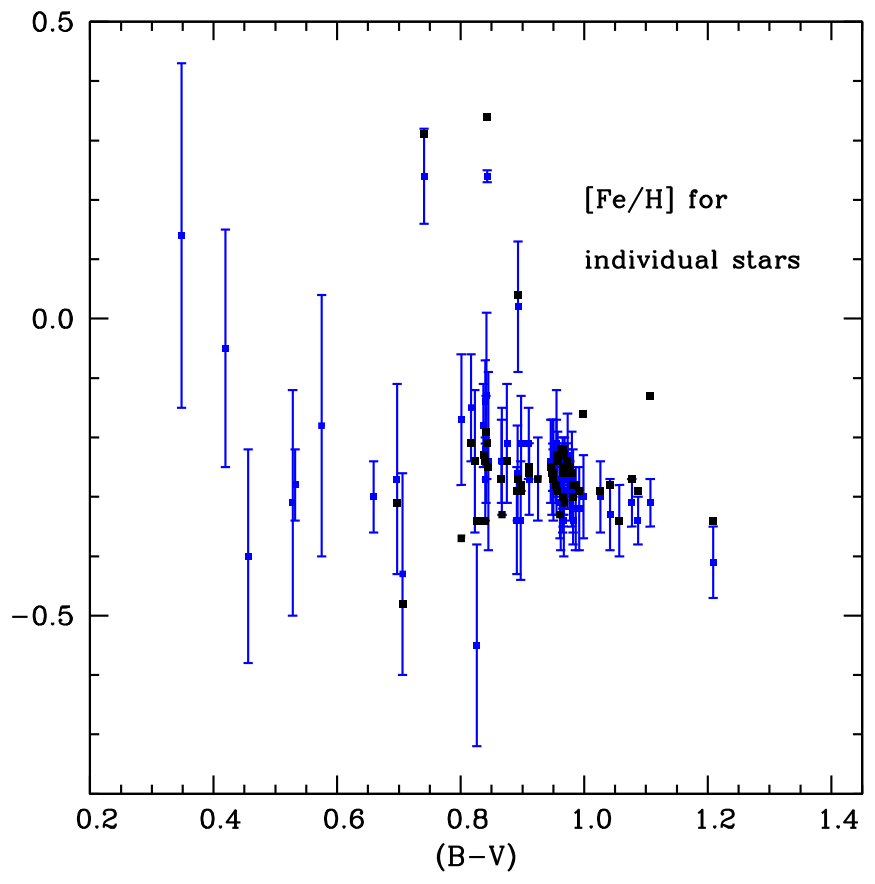

Figure 4. For each star, the median $[\mathrm{Fe} / \mathrm{H}]$ from ROBOSPECT analysis is shown as a function of $(B-V)$ color using blue symbols; the error bars indicate the size of the median absolute deviation statistic for that star. Black points designate $[\mathrm{Fe} / \mathrm{H}]$ estimates from the ANNA neural network analysis.

turnoff region is at $B-V<0.5\left(T_{\text {eff }}>6400 \mathrm{~K}\right)$; only a handful of turnoff stars remain in the analysis set and these have large uncertainties. The reason for this is the declining line strength with increasing $T_{\text {eff }}$ at a metallicity less than one-half the solar value. Thus, the statistical averages are dominated by the red giants. Among the red giants, the scatter in $[\mathrm{Fe} / \mathrm{H}]$ (Figure 4) is small, but three stars $(7041,7069,7093)$ appear to have approximately solar abundance or higher, distinctly higher than the cluster mean, error bars included. Since only radial-velocity membership is available for all three stars, it is plausible that all three are field interlopers. This is especially likely for 7069, a star located well blueward of the FRG branch at $(B-V)=0.85, V=13.56$. The other two stars fall along the subgiant branch and near the base of the giant branch. As a simple statistical check, if we plot the distribution of radial velocities for all red giants included in the study without a priori membership insight from proper motions and assume the field star histogram of Figure 1, as defined by the red giants alone, is continuous across the radial-velocity distribution range of the cluster, the predicted number of field stars with a compatible radial velocity is found to be between 2 and 5, in excellent agreement with the three metal-rich stars identified here.

We find the cluster median iron abundance to be $[\mathrm{Fe} / \mathrm{H}]=$ $-0.27 \pm 0.07 \mathrm{dex}$, where the reported uncertainty is the median absolute deviation (MAD). This value was calculated by computing the median $[\mathrm{Fe} / \mathrm{H}]$ for each star, and then using these values to compute the median/MAD $[\mathrm{Fe} / \mathrm{H}]$ for our entire sample. This reported abundance value is robust under various calculation schemes; choosing to calculate $[\mathrm{Fe} / \mathrm{H}]$ by taking the median of all $\mathrm{Fe}$ lines or by weighting each star's contribution to the overall $[\mathrm{Fe} / \mathrm{H}]$ by the number of $\mathrm{Fe}$ lines measured produces nearly the same overall result. We adopt the median/MAD statistics here as our sample size is sufficiently large and these statistics are more robust against outliers, compared with using 
the mean $/ \sigma$ statistics. In Table 3, we give the number of Fe lines measured, median and MAD for $[\mathrm{Fe} / \mathrm{H}]$, as well as estimates for $[\mathrm{Ni} / \mathrm{H}],[\mathrm{Ca} / \mathrm{H}]$, and $[\mathrm{Si} / \mathrm{H}]$ for each star in our ROBOSPECT sample. The normalized MAD statistic, $\mathrm{MADN}=1.48 \times \mathrm{MAD}$, can be used to approximate the standard deviation of our $[\mathrm{Fe} / \mathrm{H}]$ estimate. Using the MADN, we find the iron abundance for NGC 2506 to be $[\mathrm{Fe} / \mathrm{H}]=-0.27 \pm 0.013$ dex (s.e.m.). This result is the same as that generated by ANNA and only slightly higher than our photometric abundance estimate of $[\mathrm{Fe} / \mathrm{H}]=-0.32 \pm$ 0.03 (AT16).

Abundances and atmospheric parameters for each of the 65 stars with ROBOSPECT-derived abundances are reported in Table 3. For most stars we present the median abundance from 5 to $17 \mathrm{Fe}$ lines along with the MAD for that star. The $[\mathrm{Ni} / \mathrm{H}]$ abundance estimates are not based on median values for each star, since there are at most three Ni lines in our spectral region. We converted the logarithmic value for each abundance measurement to numerical values before averaging and computing the standard deviation among measurements, then converting the $\mathrm{Ni}$ abundance to a logarithmic $[\mathrm{Ni} / \mathrm{H}]$ value. The quoted error represents the effect on $[\mathrm{Ni} / \mathrm{H}]$ of a standard deviation added to the numerical $\mathrm{Ni}$ abundance value. For the sample presented in Table 3, the cluster median value of $[\mathrm{Ca} / \mathrm{H}]$ is $-0.01 \pm 0.06,[\mathrm{Si} / \mathrm{H}]=-0.33 \pm 0.05$, and $[\mathrm{Ni} / \mathrm{H}]=-0.38 \pm 0.05$. For the $\mathrm{Ca}$ and $\mathrm{Si}$ estimates, the quoted error is the MAD of all of the single-star abundance estimates relative to the cluster median value; for $\mathrm{Ni}$, the quoted error is the median value of each star's estimated error, as described above.

To get a handle on the impact of possible errors in the input parameters, the abundances were redetermined under the assumption that $T_{\text {eff }}$ was altered by $\pm 100 \mathrm{~K}, \log g$ by \pm 0.25 and $v_{t}$ by $\pm 0.25 \mathrm{~km} \mathrm{~s}^{-1}$, an approach followed in our analysis of NGC 6819 Lee-Brown et al. (2015). Our results are similar to those presented therein: the effect of altering $T_{\text {eff }}, \log g$ and $v_{t}$ by the amounts specified above is to increment the abundance $[\mathrm{Fe} / \mathrm{H}]$ by $\pm 0.06,0.00$ and $\mp 0.06$, respectively, for stars bluer than $(B-V)=0.8$. Increments for redder stars are similar: $\pm 0.06, \mp 0.03$ and $\mp 0.06$, respectively.

\subsection{Comparison to Previous Work}

There have been three studies of NGC 2506 tied to highdispersion spectroscopy (Mikolaitis et al. 2011; Reddy et al. 2012; Carlberg et al. 2016), all using red giant samples dominated by clump stars. For the three studies sampled, the overlap with the current investigation is three, two, and four stars; for Carlberg et al. (2016), star 3265 was excluded due to its peculiar nature, probably a signature of binarity. In each case, we can include two $T_{\text {eff }}\left(T_{\mathrm{NN}}\right.$ and $\left.T_{\mathrm{ph}}\right)$ and two $[\mathrm{Fe} / \mathrm{H}]$ $\left([\mathrm{Fe} / \mathrm{H}]_{\mathrm{NN}}\right.$ and $\left.[\mathrm{Fe} / \mathrm{H}]_{\mathrm{RS}}\right)$ comparisons. The $[\mathrm{Fe} / \mathrm{H}]_{\mathrm{RS}}$ refers to metallicity based upon analysis using the $T_{\mathrm{ph}}$ and EWs measured by ROBOSPECT. In all three $T_{\text {eff }}$ comparisons, the ANNA scale and the color-based scale are systematically hotter than the published values, with the color-based $T_{\text {eff }}$ always slightly hotter than the ANNA scale. The offsets for the two scales are $+24 \pm 96$ and $+66 \pm 63,+6 \pm 22$ and $+51 \pm 5$, $+19 \pm 28$ and $+63 \pm 37$, for Reddy et al. (2012), Mikolaitis et al. (2011), and Carlberg et al. (2016), respectively. The analogous offsets for $[\mathrm{Fe} / \mathrm{H}]$ from ANNA and ROBOSPECT are $-0.06 \pm 0.03$ and $-0.07 \pm 0.05,-0.03 \pm 0.07$ and $-0.01 \pm 0.04$, and $0.03 \pm 0.04$ and $0.04 \pm 0.04$, respectively.
Since the adopted $T_{\text {eff }}$ in the current investigation is hotter in all cases, transfer of the current data to the published systems will lower our $[\mathrm{Fe} / \mathrm{H}]$ in all cases, by typically $0.01-0.02 \mathrm{dex}$ for $T_{\mathrm{NN}}$ and 0.03-0.04 dex for $T_{\mathrm{ph}}$. Within the uncertainties of a small sample, this implies that we are on the same metallicity scale as Carlberg et al. (2016).

For the elements other than Fe, comparisons can be made to the discussions of Mikolaitis et al. (2011), Reddy et al. (2012). Mikolaitis et al. (2011) find [Ca/Fe], [Si/Fe], and $[\mathrm{Ni} / \mathrm{Fe}]=$ $-0.06 \pm 0.06,0.01 \pm 0.07$, and $-0.08 \pm 0.06$, respectively, while Reddy et al. (2012) get $0.10 \pm 0.05,+0.04 \pm 0.04$, and $-0.08 \pm 0.04$, respectively. Our $[\mathrm{Ca} / \mathrm{Fe}]=+0.28 \pm 0.07$ is based upon one generally strong line of $\mathrm{Ca}$ and is inconsistent with both the published spectroscopic and photometric, via the Ca II-based $h k$ index, abundances which indicate an effectively solar ratio within the uncertainties. It should be regarded as suspect. By contrast, we find $[\mathrm{Si} / \mathrm{Fe}]=-0.06 \pm 0.05$ and $[\mathrm{Ni} / \mathrm{Fe}]=-0.11 \pm 0.05$. In all three studies, $\mathrm{Si}$ is differentially more metal-rich than $\mathrm{Ni}$ by an average of $+0.09 \pm$ 0.04 dex. The dominant source of uncertainty in our ratios lies with the Si abundance, tied to a single line of modest EW in each star.

\section{Lithium \\ 5.1. Abundance Estimation}

We used the SPLOT utility within IRAF to measure EWs and Gaussian full-widths of the $\mathrm{Li} 6707.8 \AA$ line for all 287 stars. A reasonably close and relatively line-free region between $6680 \AA$ and $6690 \AA$ was examined to estimate the $\mathrm{S} / \mathrm{N}$ per pixel, also estimated using SPLOT. Our analysis of the EW measurements employs a computational scheme that first numerically removes the contribution to the $\mathrm{Li}$ line $\mathrm{EW}$ produced by the nearby $\mathrm{Fe} I$ line at $6707.45 \AA$, assuming a cluster metallicity of $[\mathrm{Fe} / \mathrm{H}]=-0.30$ and a temperature estimate for the star. For the latter input, we used the same color-temperature scheme as described earlier. The computational scheme then interpolates within a model-atmospheregenerated grid of EWs and temperatures to estimate a $\mathrm{Li}$ abundance for each star, a scheme developed by Steinhauer (2003) and employed by Steinhauer \& Deliyannis (2004). A star is only considered to have a detected $\mathrm{Li}$ abundance if the corrected EW exceeds three times the estimated error in the $\mathrm{EW}$, itself a function of the measured line width and $\mathrm{S} / \mathrm{N}$ for the spectrum (Deliyannis \& Pinsonneault 1993; Deliyannis et al. 1993). For stars with EW below this criterion, the $\mathrm{Li}$ abundance can only be characterized as an upper limit and no abundance error is estimated. The abundance error estimate for detected lines is primarily dependent on the error in the EW. We did test the sensitivity of the computed Li abundance to increments of $+100 \mathrm{~K}$ in $T_{\text {eff }}$. A higher $T_{\text {eff }}$ results in higher $\mathrm{Li}$ abundance but by widely different amounts for different classes of stars; for warmer dwarfs, the abundance increment is $\leqslant 0.1$, rising to 0.15 for cooler subgiants and high sensitivity $(\geqslant 0.3)$ for stars on the red giant branch.

A more subtle concern for the coolest stars arises from the presence of $\mathrm{CN}$ lines in the region of the Li line. From synthetic spectra, we find that at the metallicity of NGC 2506 and plausible levels of $\mathrm{CN}$ enhancement, the molecular lines have no impact on the measured EW. For stars of solar metallicity and higher, $\mathrm{CN}$-enhanced giants can be affected. 
Of the 175 stars classed as single-star members, Li detections or upper limits were possible for all but 30 . Of the 30 stars, one is 4402 , the coolest red giant member of our sample with $B-V=1.76$. The confusion and complexity of features near the $\mathrm{Li}$ line made any attempt at a $\mathrm{Li}$ abundance estimate for this star impossible. This internal photoelectric standard (McClure et al. 1981) lies outside the astrometric survey (Chiu \& van Altena 1981) and, like 2402, has generally been ignored in spectroscopic surveys of the red giant branch. Its location in the CMD, however, places this star closest to the tip of the red giant branch and a potential candidate for evidence of Li-enrichment (Carlberg et al. 2016); observation at a higher resolution could prove informative.

The 29 remaining stars fall within a well-defined group: rapidly rotating stars $\left(V_{\text {rot }}>30 \mathrm{~km} \mathrm{~s}^{-1}\right)$ at the CMD turnoff. The average measured rotation speed for the excluded stars is $54.2 \mathrm{~km} \mathrm{~s}^{-1}$, broadening the $\mathrm{Li}$ line, if one exists, to a level where, when combined with the statistical noise, any attempt at directly measuring or even placing a constraint on the $\mathrm{Li}$ value proved implausible. The $\mathrm{A}(\mathrm{Li})$ values with non-zero uncertainties for stars with measureable $\mathrm{Li}$ and zero for stars with upper limits are presented in Table 4.

\subsection{Evolution in the CMD}

For the remaining 145 stars, Figure 5 shows the CMD for stars with Li determinations; open black circles are detections while open red triangles designate upper limits. Blue symbols are detections for stars classed as subgiants, allowing one to uniquely distinguish these stars from turnoff stars of the same $B-V$ but at fainter magnitudes and from stars classed as FRGs in later figures. Filled symbols identify the three stars which are spectroscopically determined to be metal-rich and therefore likely field stars, two of which have Li detections while the third has only an upper limit. Of the 41 stars with only upper limits to $\mathrm{A}(\mathrm{Li}), 10$ are located at the turnoff (defined as $B-V<0.5$, irrespective of $V$ ) and 31 populate the giant region $(B-V>0.8)$. Among the redder stars, the sample splits into two distinct groups, stars at the base of the vertical turnup of the red giant branch and the red giant clump stars. With the exception of one anomalously blue clump star (4128), every giant located within the CMD region associated with the red clump has, at best, an upper limit for $\mathrm{A}(\mathrm{Li})$. It is crucial to note that this is not simply an issue of the clump stars exhibiting lower $B-V$ and higher than average $T_{\text {eff }}$ compared to FRGs at the same magnitude level and therefore weaker lines. The pattern among FRGs at the same $T_{\text {eff }}$ but positioned $0.5 \mathrm{mag}$ fainter than the red clump clearly demonstrates that if the clump stars had $\mathrm{A}(\mathrm{Li})$ similar to the FRGs, it should be detectable.

To probe the empirical trends among $\mathrm{A}(\mathrm{Li})$, we show in Figure 6 the variation in $\mathrm{A}(\mathrm{Li})$ as a function of $V$. Symbols have the same meaning as in Figure 5. The pattern with decreasing $V$ is a clear reflection of the evolution from the main sequence to the giant branch, with the apparent reversal between $V=15.2$ and 14.6 caused by the shape of the subgiant branch. The stars at the turnoff, irrespective of magnitude, show an approximately constant mean $\mathrm{A}(\mathrm{Li})$ near 3.05 with a range approaching $\pm 0.30 \mathrm{dex}$. $\mathrm{A}(\mathrm{Li})$ steadily declines across the subgiant branch, up to the base of the FRG branch, with every subgiant having detectable $\mathrm{Li}$, rather than just an upper limit. Between the base of the FRG at $V=14.5$ and the approximate luminosity level of the red giant clump at $V=13.0, \mathrm{~A}(\mathrm{Li})$ among the stars with detectable $\mathrm{Li}$ remains

Table 4

Lithium Abundances for NGC 2506 Stars

\begin{tabular}{|c|c|c|c|c|c|c|c|}
\hline ID No. & $T_{\text {eff }}$ & $\mathrm{EW}(\mathrm{Li})$ & $\mathrm{S} / \mathrm{N}$ & FWHM & $\mathrm{A}(\mathrm{Li})$ & $\sigma_{\mathrm{ALi}}$ & Cod \\
\hline 1108 & 5372 & 21.2 & 124 & 0.46 & 0.70 & 0.00 & 2 \\
\hline 1112 & 5070 & 16.6 & 129 & 0.80 & 0.55 & 0.00 & 3 \\
\hline 1127 & 6909 & 5.0 & 132 & 0.70 & 2.50 & 0.00 & 1 \\
\hline 1134 & 6950 & 30.5 & 158 & 1.60 & 2.94 & 0.09 & 0 \\
\hline 1211 & 7004 & 30.0 & 195 & 1.01 & 2.96 & 0.06 & 0 \\
\hline 1229 & 4856 & 53.7 & 124 & 0.67 & 1.11 & 0.06 & 0 \\
\hline 1301 & 5249 & 45.9 & 118 & 0.89 & 1.56 & 0.08 & 0 \\
\hline 1302 & 6950 & 23.0 & 117 & 0.90 & 2.80 & 0.12 & 0 \\
\hline 1305 & 7009 & 29.9 & 187 & 1.03 & 2.97 & 0.06 & 0 \\
\hline 1320 & 5051 & 13.6 & 143 & 0.64 & 0.55 & 0.00 & 3 \\
\hline 1325 & 5070 & 10.9 & 138 & 0.53 & 0.55 & 0.00 & 3 \\
\hline 1331 & 6652 & 45.4 & 155 & 0.94 & 2.94 & 0.05 & 0 \\
\hline 1340 & 5308 & 27.0 & 179 & 0.67 & 1.24 & 0.10 & 0 \\
\hline 1350 & 6936 & 56.2 & 170 & 1.94 & 3.25 & 0.05 & 0 \\
\hline 1354 & 6968 & 41.0 & 120 & 0.98 & 3.10 & 0.07 & 0 \\
\hline 1358 & 6852 & 34.6 & 198 & 2.04 & 2.94 & 0.07 & 0 \\
\hline 1377 & 5421 & 37.1 & 180 & 0.65 & 1.64 & 0.06 & 0 \\
\hline 1379 & 6959 & 27.8 & 175 & 1.10 & 2.90 & 0.07 & 0 \\
\hline 1384 & 6735 & 67.4 & 236 & 2.42 & 3.21 & 0.04 & 0 \\
\hline 1390 & 6936 & 5.0 & 140 & 0.70 & 2.49 & 0.00 & 1 \\
\hline 1394 & 6702 & 71.4 & 130 & 1.50 & 3.23 & 0.05 & 0 \\
\hline 2102 & 6847 & 44.0 & 188 & 2.30 & 3.06 & 0.07 & 0 \\
\hline 2140 & 6856 & 30.2 & 170 & 0.82 & 2.87 & 0.06 & 0 \\
\hline 2212 & 4810 & 26.1 & 96 & 0.68 & 0.40 & 0.00 & 3 \\
\hline 2217 & 6635 & 79.2 & 160 & 1.66 & 3.24 & 0.04 & 0 \\
\hline 2255 & 5063 & 39.4 & 147 & 0.72 & 1.18 & 0.08 & 0 \\
\hline 2306 & 6963 & 49.0 & 113 & 1.31 & 3.19 & 0.08 & 0 \\
\hline 2309 & 5065 & 9.4 & 145 & 0.58 & 0.55 & 0.00 & 3 \\
\hline 2311 & 6640 & 66.5 & 156 & 1.59 & 3.14 & 0.05 & 0 \\
\hline 2325 & 6968 & 5.0 & 100 & 0.70 & 2.67 & 0.00 & 1 \\
\hline 2328 & 6860 & 70.2 & 199 & 2.90 & 3.32 & 0.05 & 0 \\
\hline 2329 & 5106 & 12.4 & 156 & 0.56 & 0.55 & 0.00 & 3 \\
\hline 2332 & 6869 & 8.2 & 148 & 0.97 & 2.49 & 0.00 & 1 \\
\hline 2343 & 6945 & 32.7 & 94 & 0.70 & 2.97 & 0.09 & 0 \\
\hline 2347 & 6865 & 36.0 & 229 & 1.62 & 2.96 & 0.05 & 0 \\
\hline 2351 & 7079 & 52.2 & 250 & 1.50 & 3.30 & 0.03 & 0 \\
\hline 2364 & 5377 & 20.5 & 169 & 0.85 & 0.70 & 0.00 & 2 \\
\hline 2371 & 6927 & 32.5 & 174 & 1.02 & 2.95 & 0.06 & 0 \\
\hline 2373 & 6963 & 23.6 & 130 & 1.03 & 2.82 & 0.11 & 0 \\
\hline 2375 & 4990 & 52.2 & 146 & 0.77 & 1.29 & 0.06 & 0 \\
\hline 2380 & 5094 & 16.4 & 175 & 0.81 & 0.55 & 0.00 & 3 \\
\hline 2386 & 6945 & 39.9 & 206 & 1.25 & 3.07 & 0.05 & 0 \\
\hline 2394 & 7000 & 37.8 & 122 & 1.29 & 3.08 & 0.09 & 0 \\
\hline 2401 & 5111 & 17.4 & 163 & 0.75 & 0.55 & 0.00 & 3 \\
\hline 2402 & 4505 & 60.4 & 94 & 0.71 & 0.59 & 0.10 & 0 \\
\hline 3110 & 5364 & 25.0 & 159 & 0.68 & 1.26 & 0.13 & 0 \\
\hline 3112 & 7046 & 25.0 & 103 & 1.03 & 2.90 & 0.13 & 0 \\
\hline 3135 & 6891 & 32.8 & 115 & 1.10 & 2.94 & 0.10 & 0 \\
\hline 3158 & 6765 & 65.0 & 115 & 0.67 & 3.21 & 0.04 & 0 \\
\hline 3204 & 5231 & 9.2 & 145 & 0.66 & 0.70 & 0.00 & 3 \\
\hline 3206 & 6740 & 45.4 & 199 & 2.25 & 3.00 & 0.06 & 0 \\
\hline 3231 & 5096 & 12.4 & 142 & 0.59 & 0.55 & 0.00 & 3 \\
\hline 3263 & 6896 & 26.8 & 176 & 1.20 & 2.84 & 0.08 & 0 \\
\hline 3324 & 5089 & 15.6 & 146 & 0.62 & 0.55 & 0.00 & 3 \\
\hline 3344 & 6991 & 44.6 & 134 & 1.10 & 3.16 & 0.06 & 0 \\
\hline 3350 & 6918 & 52.7 & 174 & 1.59 & 3.20 & 0.05 & 0 \\
\hline 3356 & 5200 & 36.2 & 160 & 0.75 & 1.32 & 0.08 & 0 \\
\hline 3359 & 5106 & 12.5 & 164 & 0.61 & 0.55 & 0.00 & 3 \\
\hline 3360 & 6778 & 76.0 & 194 & 1.72 & 3.31 & 0.04 & 0 \\
\hline 3367 & 6968 & 24.2 & 160 & 1.00 & 2.84 & 0.09 & 0 \\
\hline 3373 & 6986 & 30.6 & 109 & 0.89 & 2.96 & 0.10 & 0 \\
\hline 3394 & 6927 & 5.0 & 155 & 0.70 & 2.43 & 0.00 & 1 \\
\hline 4105 & 7051 & 23.4 & 138 & 1.33 & 2.87 & 0.12 & 0 \\
\hline 4106 & 6887 & 5.0 & 157 & 0.70 & 2.40 & 0.00 & 1 \\
\hline
\end{tabular}


Table 4

(Continued)

\begin{tabular}{|c|c|c|c|c|c|c|c|}
\hline ID No. & $T_{\text {eff }}$ & $\mathrm{EW}(\mathrm{Li})$ & $\mathrm{S} / \mathrm{N}$ & FWHM & $\mathrm{A}(\mathrm{Li})$ & $\sigma_{\mathrm{ALi}}$ & Cod \\
\hline 4109 & 5311 & 31.8 & 180 & 0.68 & 1.38 & 0.08 & 0 \\
\hline 4128 & 5246 & 61.4 & 177 & 0.67 & 1.75 & 0.03 & 0 \\
\hline 4129 & 6314 & 64.6 & 118 & 0.98 & 2.88 & 0.05 & 0 \\
\hline 4138 & 5094 & 20.6 & 172 & 0.82 & 0.55 & 0.00 & 2 \\
\hline 4145 & 6959 & 21.8 & 120 & 0.88 & 2.78 & 0.12 & 0 \\
\hline 4205 & 5094 & 19.7 & 162 & 0.90 & 0.55 & 0.00 & 2 \\
\hline 4216 & 6731 & 74.8 & 195 & 1.56 & 3.27 & 0.04 & 0 \\
\hline 4218 & 7327 & 30.6 & 161 & 0.98 & 3.18 & 0.07 & 0 \\
\hline 4223 & 5886 & 60.8 & 200 & 0.77 & 2.48 & 0.03 & 0 \\
\hline 4240 & 5030 & 13.5 & 119 & 0.70 & 0.55 & 0.00 & 3 \\
\hline 4274 & 5035 & 37.4 & 160 & 0.92 & 1.09 & 0.09 & 0 \\
\hline 4331 & 6883 & 61.4 & 198 & 2.78 & 3.26 & 0.05 & 0 \\
\hline 4337 & 6575 & 84.4 & 170 & 1.88 & 3.24 & 0.04 & 0 \\
\hline 4353 & 6968 & 26.8 & 145 & 0.99 & 2.89 & 0.09 & 0 \\
\hline 4361 & 6635 & 43.2 & 213 & 1.88 & 2.90 & 0.05 & 0 \\
\hline 4372 & 6607 & 53.1 & 188 & 1.13 & 2.99 & 0.04 & 0 \\
\hline 4528 & 5374 & 38.8 & 160 & 0.69 & 1.62 & 0.06 & 0 \\
\hline 5196 & 6977 & 32.0 & 148 & 1.35 & 2.98 & 0.08 & 0 \\
\hline 5214 & 6453 & 69.5 & 208 & 1.50 & 3.03 & 0.03 & 0 \\
\hline 5233 & 6909 & 58.2 & 190 & 1.61 & 3.25 & 0.04 & 0 \\
\hline 5249 & 5413 & 28.8 & 145 & 0.77 & 1.46 & 0.10 & 0 \\
\hline 5270 & 7600 & 5.0 & 230 & 0.70 & 2.69 & 0.00 & 1 \\
\hline 5271 & 5108 & 13.5 & 144 & 0.65 & 0.55 & 0.00 & 3 \\
\hline 5371 & 5385 & 5.0 & 135 & 0.70 & 0.70 & 0.00 & 3 \\
\hline 7008 & 5234 & 19.3 & 138 & 0.97 & 0.70 & 0.00 & 3 \\
\hline 7015 & 6847 & 41.4 & 180 & 1.58 & 3.02 & 0.06 & 0 \\
\hline 7019 & 5202 & 33.7 & 159 & 0.64 & 1.27 & 0.07 & 0 \\
\hline 7022 & 6852 & 49.0 & 200 & 3.82 & 3.11 & 0.07 & 0 \\
\hline 7023 & 5068 & 17.3 & 159 & 0.63 & 0.55 & 0.00 & 3 \\
\hline 7026 & 5065 & 38.8 & 145 & 0.67 & 1.17 & 0.08 & 0 \\
\hline 7031 & 5744 & 76.0 & 200 & 0.89 & 2.47 & 0.03 & 0 \\
\hline 7032 & 6161 & 74.2 & 193 & 0.99 & 2.84 & 0.03 & 0 \\
\hline 7035 & 6472 & 71.6 & 165 & 0.99 & 3.07 & 0.03 & 0 \\
\hline 7036 & 5051 & 18.3 & 129 & 0.59 & 0.55 & 0.00 & 3 \\
\hline 7038 & 6786 & 67.8 & 165 & 2.44 & 3.25 & 0.06 & 0 \\
\hline 7041 & 5643 & 66.2 & 129 & 0.77 & 2.27 & 0.04 & 0 \\
\hline 7042 & 6765 & 55.4 & 185 & 1.46 & 3.12 & 0.04 & 0 \\
\hline 7043 & 5377 & 46.4 & 124 & 0.78 & 1.74 & 0.07 & 0 \\
\hline 7045 & 4741 & 21.9 & 89 & 0.63 & 0.20 & 0.00 & 3 \\
\hline 7047 & 6968 & 36.0 & 140 & 1.12 & 3.03 & 0.07 & 0 \\
\hline 7048 & 5021 & 17.9 & 150 & 0.84 & 0.55 & 0.00 & 3 \\
\hline 7053 & 6896 & 46.7 & 177 & 1.21 & 3.12 & 0.05 & 0 \\
\hline 7058 & 6932 & 30.3 & 151 & 1.10 & 2.92 & 0.08 & 0 \\
\hline 7059 & 6923 & 65.6 & 173 & 3.24 & 3.32 & 0.06 & 0 \\
\hline 7061 & 6689 & 6.0 & 198 & 0.70 & 2.16 & 0.00 & 1 \\
\hline 7062 & 6830 & 5.0 & 230 & 0.70 & 2.18 & 0.00 & 1 \\
\hline 7063 & 6744 & 51.0 & 195 & 2.01 & 3.06 & 0.05 & 0 \\
\hline 7064 & 6941 & 40.5 & 157 & 1.16 & 3.07 & 0.06 & 0 \\
\hline 7066 & 6761 & 9.0 & 261 & 0.50 & 2.23 & 0.10 & 0 \\
\hline 7069 & 5369 & 30.7 & 105 & 0.75 & 1.44 & 0.14 & 0 \\
\hline 7071 & 7107 & 34.6 & 125 & 1.80 & 3.10 & 0.11 & 0 \\
\hline 7076 & 6808 & 48.2 & 125 & 1.55 & 3.08 & 0.08 & 0 \\
\hline 7080 & 5288 & 26.4 & 186 & 0.64 & 1.19 & 0.10 & 0 \\
\hline 7081 & 6905 & 24.4 & 164 & 0.86 & 2.80 & 0.08 & 0 \\
\hline 7082 & 5771 & 54.7 & 189 & 0.68 & 2.29 & 0.03 & 0 \\
\hline 7084 & 4787 & 28.3 & 120 & 0.64 & 0.20 & 0.00 & 2 \\
\hline 7085 & 4928 & 55.6 & 168 & 0.77 & 1.25 & 0.05 & 0 \\
\hline 7086 & 5479 & 51.9 & 140 & 0.95 & 1.93 & 0.06 & 0 \\
\hline 7088 & 6329 & 73.0 & 156 & 0.87 & 2.97 & 0.03 & 0 \\
\hline 7089 & 6673 & 66.0 & 242 & 2.71 & 3.16 & 0.04 & 0 \\
\hline 7090 & 6950 & 45.4 & 162 & 1.11 & 3.14 & 0.05 & 0 \\
\hline 7093 & 5244 & 25.4 & 124 & 0.98 & 0.70 & 0.00 & 2 \\
\hline 7094 & 6778 & 49.0 & 193 & 1.18 & 3.06 & 0.04 & 0 \\
\hline 7 & 6619 & 8 & 201 & 1.82 & 2.84 & 0.06 & \\
\hline
\end{tabular}

Table 4

(Continued)

\begin{tabular}{lccccccc}
\hline \hline ID No. & $T_{\text {eff }}$ & EW(Li) & $\mathrm{S} / \mathrm{N}$ & FWHM & $\mathrm{A}(\mathrm{Li})$ & $\sigma_{\mathrm{ALi}}$ & Code \\
\hline 7098 & 5030 & 10.2 & 176 & 0.69 & 0.55 & 0.00 & 3 \\
7099 & 5042 & 20.4 & 146 & 0.82 & 0.55 & 0.00 & 2 \\
7102 & 5070 & 17.0 & 156 & 0.78 & 0.55 & 0.00 & 3 \\
7104 & 6757 & 46.6 & 192 & 1.38 & 3.02 & 0.05 & 0 \\
7105 & 6918 & 67.7 & 143 & 1.73 & 3.34 & 0.05 & 0 \\
7106 & 4891 & 55.0 & 127 & 0.69 & 1.19 & 0.06 & 0 \\
7108 & 5007 & 41.8 & 155 & 0.73 & 1.15 & 0.07 & 0 \\
7109 & 6900 & 24.6 & 132 & 1.42 & 2.80 & 0.12 & 0 \\
7110 & 6923 & 5.0 & 118 & 0.70 & 2.56 & 0.00 & 1 \\
7111 & 6972 & 16.1 & 191 & 1.09 & 2.65 & 0.11 & 0 \\
7114 & 5080 & 12.8 & 180 & 0.64 & 0.55 & 0.00 & 3 \\
7115 & 7102 & 32.0 & 116 & 0.61 & 3.06 & 0.07 & 0 \\
7117 & 5051 & 22.8 & 165 & 0.79 & 0.55 & 0.00 & 2 \\
7123 & 6727 & 58.2 & 232 & 0.91 & 3.12 & 0.03 & 0 \\
7125 & 6865 & 24.8 & 155 & 1.20 & 2.78 & 0.09 & 0 \\
7128 & 5115 & 39.7 & 180 & 0.76 & 1.27 & 0.06 & 0 \\
7132 & 6843 & 62.8 & 169 & 2.18 & 3.24 & 0.05 & 0 \\
\hline
\end{tabular}

Note. Lithium abundance values with non-zero errors (code 0 ) are detections; stars with abundance codes 1,2 or 3 are upper limits constrained by $\mathrm{S} / \mathrm{N}$, the temperature grid, or both, respectively.

(This table is available in machine-readable form.)

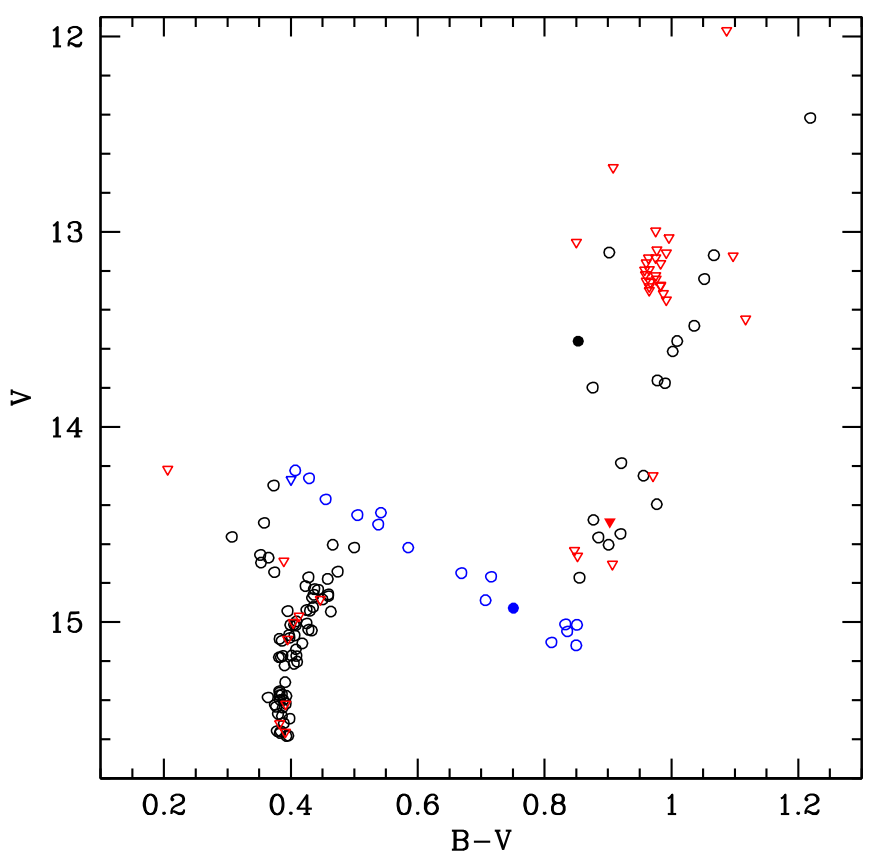

Figure 5. CMD for single-star members with detectable Li (open black circles) and upper limits to $\mathrm{A}(\mathrm{Li})$ (open red triangles). Blue symbols identify the location of purported members of the subgiant branch. Filled symbols identify the three stars with estimated metallicities significantly higher than the cluster.

effectively constant near $\mathrm{A}(\mathrm{Li})=1.25$. Only one star (2402) above the clump level has measurable $\mathrm{Li}$, with $\mathrm{A}(\mathrm{Li})$ just below 0.6 . As a newly identified probable member of the cluster, this star plays a unique role in redefining the location of the luminous end of the FRG branch. Previous discussions (Carretta et al. 2004; Mikolaitis et al. 2011; Reddy et al. 2012) have assumed that the FRG branch above the clump passed through the positions occupied by stars 2212 ( $(B-$ $V)=1.09, V=11.97)$ and $2122(1.10,11.7)$ (not included in 


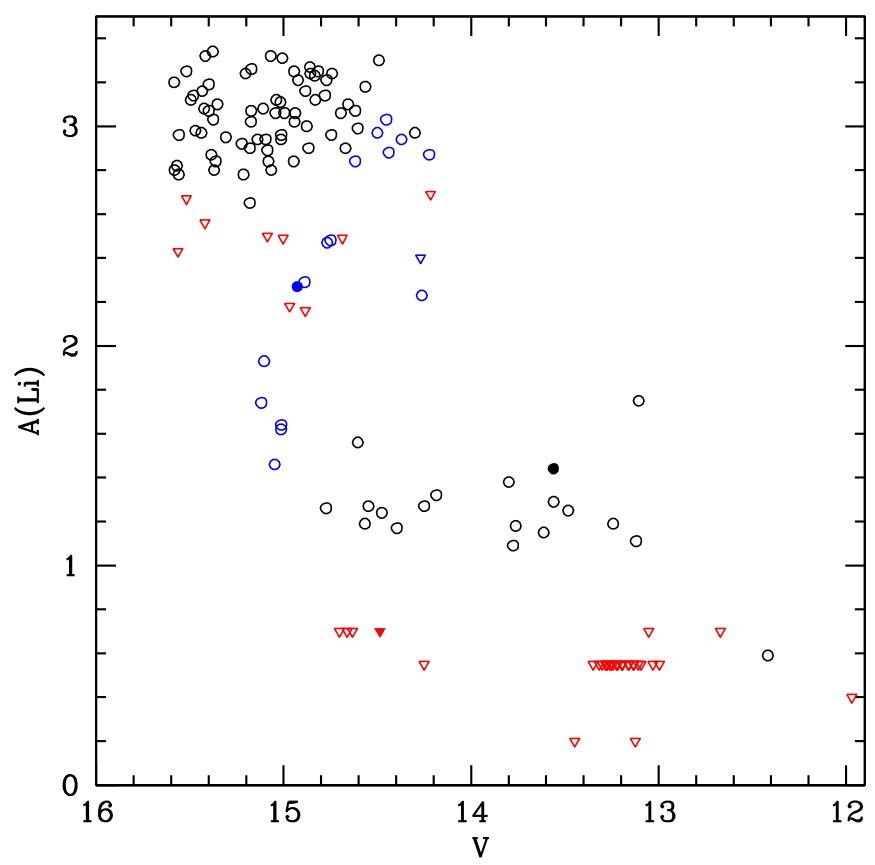

Figure 6. Li abundance as a function of $V$. Symbols have the same meaning as in Figure 5.

the figure). The former star, included in this analysis, only has an upper limit to $\mathrm{A}(\mathrm{Li})$, as expected if it is a post-He-flash star. (But see the discussion on star 4128 , below, on the possibility of $\mathrm{Li}$ production by post-He-flash stars.) If 2402 is truly a cluster member defining the FRG branch, then 2212 and 2122 lie almost a magnitude above this extension and cannot be normal, single stars in this same phase of evolution, i.e., they cannot be FRGs.

The fact that $\mathrm{Li}$ is detectable in 2402 at a level comparable to that found as an upper limit among the bluer and fainter clump stars is due in part to its having the coolest $T_{\text {eff }}$ of any star with a measurable spectrum included in the membership sample. However, if its proposed evolutionary state is correct, it could be evidence that $\mathrm{A}(\mathrm{Li})$ does decline among FRGs beyond the level of the clump and prior to the He-flash, a point returned to below.

One star (4128) near $V=13.1$ has $\mathrm{A}(\mathrm{Li})=1.75$, higher than the average of 1.25 for FRG stars over a range in luminosity. It is located in Figure 5 blueward of the red giant clump, leading to potential classification as a Li-rich giant since it sits just above the relatively fluid boundary used to define such stars (e.g., Kumar et al. 2011; Silva Aguirre et al. 2014; Casey et al. 2016; Takeda \& Tajitsu 2017). From isochrone comparisons (VandenBerg et al. 2006), the initial mass of the stars populating the giant branch in NGC 2506 is between 1.6 and $1.7 M_{\odot} . \mathrm{A}(\mathrm{Li}) \sim 1.5$ is what standard models predict for a star of $1.5 M_{\odot}$ and solar abundance after the first dredge-up (FDU; Palmerini et al. 2011), but not for post He-flash red giants. Empirically, however, the star has $\mathrm{A}(\mathrm{Li}) \sim 0.5 \mathrm{dex}$ above the observed value for the FRGs, irrespective of the predictions of standard models, which may be a signature of actual $\mathrm{Li}$ production in a star in the post-He-core-flash phase (Monaco et al. 2014; Silva Aguirre et al. 2014). No other star on the giant branch, above or below the clump, exhibits $\mathrm{Li}$ sufficiently high enough to be tagged as a potential Li-rich star, though this is not unexpected given the often quoted $1 \%$ rate of detection for these stars in the general population of red giants.

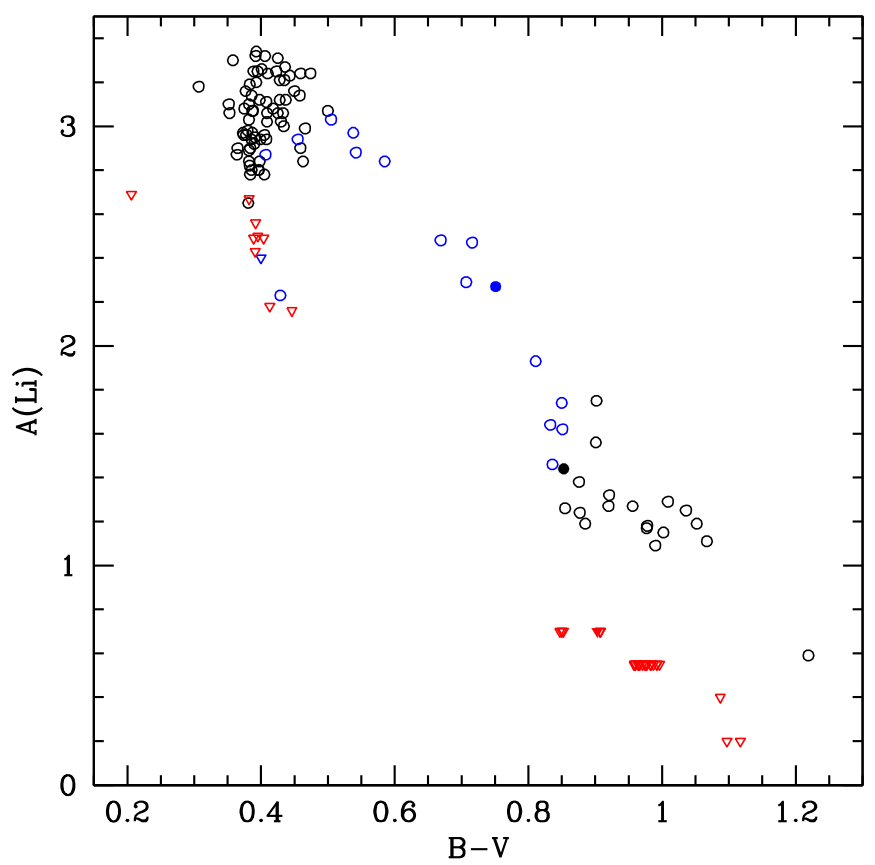

Figure 7. $\mathrm{Li}$ abundance as a function of $B-V$. Symbols have the same meaning as in Figure 5.

A clearer vision of the evolutionary path is shown in Figure 7, where $\mathrm{A}(\mathrm{Li})$ is plotted as a function of the $B-V$ color. For stars at the turnoff, there is again no trend of measured $\mathrm{A}(\mathrm{Li})$ with color between $B-V=0.3$ and 0.5 . However, once the stars initiate evolution across the subgiant branch, there is a steady decline to a typical detection value near $\mathrm{A}(\mathrm{Li}) \sim 1.25$, followed by no significant decline from the base of the giant branch to the level of the clump. With the exception of only 4128 , all stars readily identifiable as post-Heflash red giants exhibit only upper limits to $\mathrm{A}(\mathrm{Li})$.

What can we learn from these patterns, especially in light of the predictions from standard stellar evolution models? Beginning with the turnoff, there are 72 stars with $B-V \leqslant$ 0.50 with detectable $\mathrm{Li}$. The average $\mathrm{A}(\mathrm{Li})$ for these stars is $3.04 \pm 0.19$ (s.d.) dex; if one extreme outlier with $\mathrm{A}(\mathrm{Li})=$ 2.23 is excluded, the average becomes $3.05 \pm 0.16$ (s.d.); the dispersion is more than double the value of 0.07 derived from the average standard deviation as defined by the precision of the individual measures. The stars at the turnoff of NGC 2506 started on the unevolved main sequence hotter than the Li-dip, which is located at a fainter $V$ than accessed by the spectroscopic sample. Under standard stellar evolution models, the turnoff stars should retain their initial $\mathrm{A}(\mathrm{Li})$ until they evolve to $T_{\text {eff }}<5600 \mathrm{~K}$, the site of the first signature of the FDU phase, equivalent to $(B-V) \sim 0.75$ for the subgiant stars in NGC 2506, i.e., until one reaches the reddest stars on the subgiant branch (blue symbols) at the base of the vertical FRG branch (e.g., Pinsonneault 1997; Charbonnel \& Lagarde 2010).

\subsection{Initial Conditions: The Primordial Cluster Li Abundance}

While it is generally agreed that the location of the Li-dip is purely temperature-dependent, leading to mass ranges for the Li-dip strongly correlated with $[\mathrm{Fe} / \mathrm{H}]$ (Balachandran 1995; Chen et al. 2001; Anthony-Twarog et al. 2009; Cummings et al. 2012; Ramírez et al. 2012), a distinctly different question 
is the evolution of the $\mathrm{Li}$ abundance with time and $[\mathrm{Fe} / \mathrm{H}]$ within the Galaxy. Did clusters with lower $[\mathrm{Fe} / \mathrm{H}]$ form with a lower initial $\mathrm{A}(\mathrm{Li})$ or is the time of formation the determining factor? This question is independent of the discrepancy between the $\mathrm{Li}$ abundance among globular cluster stars and the primordial estimate from cosmology (Coc et al. 2014) since both lie at $\mathrm{A}(\mathrm{Li})=2.7$ or less, well below the solar system (Asplund et al. 2009) and young cluster estimate of $\sim 3.3$ (Jones et al. 1997; Steinhauer \& Deliyannis 2004; Balachandran et al. 2010; Cummings et al. 2017). The relevance for the current discussion is that the determination of the physical processes producing a spread of $0.6 \mathrm{dex}$ in $\mathrm{A}(\mathrm{Li})$ among the stars at the turnoff will be heavily weighted by whether the current observed upper bound in $\mathrm{A}(\mathrm{Li})$ was the initial cluster value and all deviations from that value are due to Li depletions or the primordial cluster value was lower and the spread is a combination of both enhancements and depletions of Li relative to the mean.

The difficulty in defining the original cluster value arises from the fact that for stars within the Li-dip and cooler, $\mathrm{A}(\mathrm{Li})$ declines over time once stars have reached the main sequence. While the rate of decline will vary with $T_{\text {eff }}$, with hotter stars on the red side of the Li-dip depleting at a slower rate, unless stars are observed relatively soon after attaining the main sequence, the observed abundance supplies only a lower bound. This approach has been adopted by investigations attempting to link globular cluster Li observations to the disk clusters and field stars as in Dobrovolskas et al. (2014) or from analyses of field star samples of the thin and thick disk, as recently exemplified by Fu et al. (2017). While such discussions invariably conclude that $\mathrm{A}(\mathrm{Li})$ has grown between the formation of the metaldeficient globulars and the current disk, the relative role of age versus metallicity in defining the growth remains obscure. As already noted, open cluster studies demonstrate that $\mathrm{A}(\mathrm{Li})$ depletion for stars cooler than $6500 \mathrm{~K}$ is a strong function of temperature and age, even for stars populating the $\mathrm{Li}$ plateau redward of the Li-dip (Cummings et al. 2012). The discussion by $\mathrm{Fu}$ et al. (2017), as an example, derives the trend of $\mathrm{A}(\mathrm{Li})$ with $[\mathrm{Fe} / \mathrm{H}]$ by averaging the six most Li-rich stars in each metallicity bin from a sample of $\sim 300$ stars ranging in $[\mathrm{Fe} / \mathrm{H}]$ from -1 to +0.5 , sorted into thick and thin disk components based upon $[\alpha / \mathrm{Fe}]$ as a function of $[\mathrm{Fe} / \mathrm{H}]$. The stars with the highest measured $\mathrm{A}(\mathrm{Li})$ come from a $T_{\text {eff }}$ range of 6500 to $\sim 5500 \mathrm{~K}$. The sample includes stars from the unevolved main sequence to the subgiant branch. Without knowing (a) the selection biases within the original sample, (b) the sensitivity of the results to the exact choice of the thick/thin disk boundary, a strong function of $[\mathrm{Fe} / \mathrm{H}]$, and (c) the exact $T_{\text {eff }}$ and age distribution for each star used in defining the upper bound with age, the significance of their derived trend remains questionable. Since no $[\mathrm{Fe} / \mathrm{H}]$ bin attains an $\mathrm{A}(\mathrm{Li})$ upper limit above 2.85 , it is clear that the dominant majority of the sample has depleted $\mathrm{A}(\mathrm{Li})$ from whatever value they formed with to their current level and attempting to predict the original value which defines the trend with age or $[\mathrm{Fe} / \mathrm{H}]$ becomes a futile and inherently biased exercise.

From standard stellar evolution theory (SSET), the solution to this constraint would be to observe stars hotter than the Lidip since these stars should undergo no depletion because their convective atmospheres are thin to non-existent.

The obvious problem with this approach to testing the evolution of $\mathrm{A}(\mathrm{Li})$ with age in the Galaxy is that, except for star clusters with metallicities well below solar, by an age of 3-4 Gyr the majority of stars hotter than the Li-dip have evolved off the main sequence and Li-dip stars are populating the turnoff region, as illustrated by M67 and NGC 6253 (Cummings et al. 2012) and Ber 32 (Randich et al. 2009). This clearly mass-dependent transition from stars with normal Li abundance on the main sequence to those which are guaranteed to leave the main sequence already exceptionally depleted in $\mathrm{Li}$ makes comparisons between the giant branches of clusters younger than $\sim 2.5 \mathrm{Gyr}$ with those older than this (the exact boundary is, again, metallicity-dependent) generally meaningless without first renormalizing the $\mathrm{Li}$ scale for the older, lower-mass stars.

A cluster which is more metal-poor than NGC 2506 with $[\mathrm{Fe} / \mathrm{H}] \sim-0.5$ and somewhat older $(3-4 \mathrm{Gyr})$ is NGC 2243 (Anthony-Twarog et al. 2005; VandenBerg et al. 2006; Jacobson et al. 2011; Francois et al. 2013). Unfortunately, the cluster sits tantalizing close to the boundary where the hot edge of the Li-dip is barely contained within the turnoff region. The majority of the stars with published A(Li) (Hill \& Pasquini 2000; Francois et al. 2013) approaching the top of the turnoff have only upper limits to their $\mathrm{A}(\mathrm{Li})$, as expected for Li-dip stars. There are, however, five stars extending toward the subgiant branch which have measurable $\mathrm{Li}$, with values ranging from $\mathrm{A}(\mathrm{Li})=2.92$ to 2.39 , leading Francois et al. (2013) to adopt $\mathrm{A}(\mathrm{Li})=2.7 \pm 0.2$ as the primordial $\mathrm{Li}$ abundance of the cluster. If, instead, the two largest values are adopted as indicative of the original cluster abundance, $\mathrm{A}(\mathrm{Li})=2.9$. Preliminary analysis of a much larger sample of turnoff and subgiant spectra obtained with HYDRA during the same observing cycle as NGC 2506 extends the detection limit for turnoff members of NGC 2243 to A(Li) above 3.0, with the majority of stars covering the range between 2.8 and 2.3. Statistical issues aside, since NGC 2243 is both older and more metal-poor than NGC 2506, the apparent reduced Li boundary for the hot side of the turnoff does not supply any insight into which parameter, time of formation or metallicity, has greater influence on the primordial $\mathrm{A}(\mathrm{Li})$ value.

A similar problem applies to the more metal-rich ([Fe/ $\mathrm{H}]=-0.02$ Lee-Brown et al. 2015) cluster, NGC 6819, with an age of $2.25 \mathrm{Gyr}$ (C. P. Deliyannis et al. 2018, in preparation). Although it is much closer in age to NGC 2506 than NGC 2243, the higher metallicity places the Li-dip at a higher mass than in NGC 2506, and thus the stars brighter than the Li-dip are, on average, more evolved than the stars brighter than the Li-dip in NGC 2506. The apparent result is that the stars brighter than the Li-dip at the turnoff in NGC 6819 exhibit a much wider range of $\mathrm{A}(\mathrm{Li})$ than found among the turnoff stars in NGC 2506. As we will discuss below, the significant spread, from an upper limit for single stars of $\mathrm{A}(\mathrm{Li})=3.2$ to detections below $\mathrm{A}(\mathrm{Li})=2.0$, and non-detections for a majority of the stars, is a clear indication that some parameter other than $T_{\text {eff }}$ must serve as a catalyst for Li-depletion upon leaving the main sequence.

Perhaps the best analog to NGC 2506 is IC 4651, a cluster consistently observed through both photometry and spectroscopy to be above solar metallicity with $[\mathrm{Fe} / \mathrm{H}]$ typically between +0.10 and +0.15 (Anthony-Twarog \& Twarog 2000; Meibom et al. 2002; Carretta et al. 2004; Pasquini et al. 2004; Santos et al. 2009). Its younger age than NGC 2506 compensates in part for the shift to higher mass for the location of the Li-dip, placing the stars near the turnoff in a position relative to the 
Li-dip similar to that of NGC 2506. The turnoff region of the cluster is tight and well-defined, as in NGC 2506, and it is one of the few clusters in the $1-2$ Gyr age range to have a few stars populate the subgiant branch just beyond the turnoff. While the sample of stars observed for $\mathrm{Li}$ (and $\mathrm{Be}$ ) to date is modest (Balachandran et al. 1991; Pasquini et al. 2004; Smiljanic et al. 2010), the composite Li data for the cluster at higher mass than the Li-dip show a maximum $\mathrm{A}(\mathrm{Li})$ between 3.3 and 3.4 before dropping precipitously across the subgiant branch (see Figure 9 of Anthony-Twarog et al. 2009). Unfortunately, the full range of stars at the turnoff brighter than the Li-dip extends down to $\mathrm{A}(\mathrm{Li}) \sim 2.5$, a spread confirmed using the composite sample from NGC 752, NGC 3680, and IC 4651 (AnthonyTwarog et al. 2009).

Looking to clusters younger and more metal-rich than NGC 2506, the obvious choices are the virtually identical clusters, the Hyades and Praesepe, as recently investigated by Cummings et al. (2017). With $[\mathrm{Fe} / \mathrm{H}]=+0.15$ and ages less than $1 \mathrm{Gyr}$, the combined sample should provide a reasonable test of any correlation between $\mathrm{A}(\mathrm{Li})$ and $[\mathrm{Fe} / \mathrm{H}]$ at a given $T_{\text {eff }}$ or main sequence mass. As illustrated in Figure 13 of Cummings et al. (2017), from the small composite sample using only single-star cluster members, $\mathrm{A}(\mathrm{Li})$ does rise to a plateau value of $\mathrm{A}(\mathrm{Li}) \sim 3.3$ just hotter than the Li-dip boundary. Among the hotter A stars, however, the Li abundance declines, reaching $\mathrm{A}(\mathrm{Li}) \sim 2.5$.

Perhaps the best evidence for a $\mathrm{Li}-\mathrm{Fe}$ correlation comes from an analysis of Hyades-age clusters ranging in $[\mathrm{Fe} / \mathrm{H}]$ from +0.15 to -0.23 by Cummings (2011). In addition to the already mentioned Hyades and Praesepe sample at $[\mathrm{Fe} / \mathrm{H}]=$ $+0.15, \mathrm{~A}(\mathrm{Li})$ as a function of $T_{\text {eff }}$ from $\sim 7000$ to $4500 \mathrm{~K}$ was measured for a large sample of stars in NGC $2539([\mathrm{Fe} / \mathrm{H}]=$ $0.00)$, IC $4756([\mathrm{Fe} / \mathrm{H}]=-0.10), \quad \mathrm{NGC} 6633([\mathrm{Fe} / \mathrm{H}]=$ $-0.10)$, and $\mathrm{M} 36[\mathrm{Fe} / \mathrm{H}]=-0.23$. Each cluster exhibited a well-defined trend between $\mathrm{A}(\mathrm{Li})$ and $T_{\text {eff }}$, with virtually identical patterns for paired clusters of the same metallicity. However, when the relations at the three metallicities were superposed, they had distinctly different zero-points and slopes. For $T_{\text {eff }}>5700 \mathrm{~K}, \mathrm{~A}(\mathrm{Li})$ declined with decreasing $[\mathrm{Fe} / \mathrm{H}]$ while for the cooler sample, the pattern reversed. The solution to the contradiction with the theoretical prediction from main sequence models with mixing due to convection is twofold: first, adopt a primordial cluster A(Li) strongly correlated with $[\mathrm{Fe} / \mathrm{H}]$, i.e., the Hyades and Praesepe formed with $\mathrm{A}(\mathrm{Li})$ higher than that found in IC 4756 and NGC 6633 by $~ 0.3$ dex. Based upon the relation derived from cluster data (Cummings 2011), a straightforward, unweighted linear fit between $\mathrm{A}(\mathrm{Li})$ and $[\mathrm{Fe} / \mathrm{H}]$ over the $[\mathrm{Fe} / \mathrm{H}]$ range from -0.2 to +0.1 gives:

$$
\mathrm{A}(\mathrm{Li})=3.315( \pm 0.003)+0.959( \pm 0.034)[\mathrm{Fe} / \mathrm{H}] .
$$

One would expect a cluster with $[\mathrm{Fe} / \mathrm{H}]=-0.27$ like NGC 2506 to have a primordial value of $\mathrm{A}(\mathrm{Li})=3.06$, consistent with the mean value observed among the turnoff stars more massive than the Li-dip. Second, for stars cooler than the Lidip, the rate of $\mathrm{Li}$ destruction at a given $T_{\text {eff }}$ is metallicity dependent, with more metal-rich clusters exhibiting higher rates of depletion.

Collectively, the evidence points (a) with high probability to the fact that the scatter in $\mathrm{A}(\mathrm{Li})$ among the stars at the cluster turnoff is real and a product of evolution on the main sequence and (b) to the possibility that the lower mean cluster Li abundance in NGC $2506(\mathrm{~A}(\mathrm{Li})=3.0)$ compared with clusters with more Hyades-like metallicity $(\mathrm{A}(\mathrm{Li})=3.4)$ is real and is tied to the intrinsically lower $[\mathrm{Fe} / \mathrm{H}]$ of the former. Just how much NGC 2506 and NGC 2243 lie below the more metal-rich objects ultimately depends on how one weights the wide distribution of $\mathrm{A}(\mathrm{Li})$ found among the sample of stars more massive than the Li-dip when deriving the mean $\mathrm{A}(\mathrm{Li})$. Particularly for clusters older than the Hyades, there is growing evidence that $\mathrm{A}(\mathrm{Li})$ above the Li-dip shows a more extensive range, especially toward lower $\mathrm{A}(\mathrm{Li})$, as a cluster ages $(\mathrm{C}$. $\mathrm{P}$. Deliyannis et al. 2018, in preparation). Equally concerning is the observational fact that the range in $\mathrm{A}(\mathrm{Li})$ for these samples, irrespective of the cluster $[\mathrm{Fe} / \mathrm{H}]$, exhibits a similar upper bound approaching $\mathrm{A}(\mathrm{Li})=3.35$. We will return to this point after discussing the trends in $\mathrm{A}(\mathrm{Li})$ among stars beyond the cluster turnoff.

\subsection{Evolution on the Subgiant Branch and Beyond}

The second empirical insight gained from the $\mathrm{A}(\mathrm{Li})$ measures comes from the color evolution illustrated in Figure 7. There is a clear range of $\mathrm{A}(\mathrm{Li})$ between 2.75 and 3.35 for the stars at the turnoff with $B-V$ bluer than 0.5 but no statistical evidence for a variation with temperature/color or $V$. For stars with $B-V$ redder than 0.5 , there is a clear decline in $\mathrm{A}(\mathrm{Li})$ as stars evolve across the subgiant branch, reaching a typical abundance of $\mathrm{A}(\mathrm{Li}) \sim 1.25$ at the base of the red giant branch, near the expected location for the end of the FDU phase. The pattern of a distinct transition in $\mathrm{A}(\mathrm{Li})$ as a function of $T_{\text {eff }}$ is reminiscent of the Li-dip on the blue/hotter side among less evolved main sequence stars. From the precision composite data for young clusters (Cummings et al. 2017), intermediate-age clusters (Anthony-Twarog et al. 2009; Cummings et al. 2012), and field stars (Ramírez et al. 2012), stars on the unevolved main sequence which were hotter than $T_{\text {eff }} \sim 6700 \mathrm{~K}$ exhibit well-defined values of $\mathrm{A}(\mathrm{Li})$ between 3.2 and 3.4 or, at minimum, a range of $\mathrm{A}(\mathrm{Li})$ extending as high as these limiting values. For stars at $T_{\text {eff }} \sim 6600 \mathrm{~K}$ and as old as or older than the Hyades, one can only find stars with minimal or undetectable $\mathrm{A}(\mathrm{Li})$, defining the approximate center of the Li-dip. This $T_{\text {eff }}$ boundary is readily seen in intermediateage and older clusters where the unevolved main sequence temperature pattern translates into a break at a specific $V$ magnitude due to the evolution of the key mass range into an approximately vertical turnoff (see, e.g., Figure 10 of AnthonyTwarog et al. 2009).

If the physical mechanism defining the high-mass boundary of the Li-dip is predominantly defined by the $T_{\text {eff }}$ of the star, stars of higher mass which form with temperatures hotter than this limit might be expected to initiate Li-depletion upon crossing this boundary during post-main-sequence evolution toward and across the subgiant branch. For stars at the turnoff of NGC 2506, with $E(B-V)=0.06$, the color boundary for $T_{\text {eff }}=6700 \mathrm{~K}$ should be $B-V=0.43$. While the sample is small, the majority of stars between $B-V=0.43$ and 0.5 do not show a dramatic drop in $\mathrm{A}(\mathrm{Li})$; the boundary of $B-$ $V=0.5$ is equivalent to $T_{\text {eff }}=6450 \mathrm{~K}$, placing it already beyond the center of the Li-dip defined by lower mass stars. In short, dwarfs exhibit a distinct Li-dip while higher-mass subgiants in the same $T_{\text {eff }}$ range do not. Since the Li-dip among dwarfs requires a few hundred million years to develop, the lack of a distinct boundary could arise from the more rapid evolutionary timescale for stars crossing the subgiant branch. This immediate lack of coupling by stars at the turnoff and the 


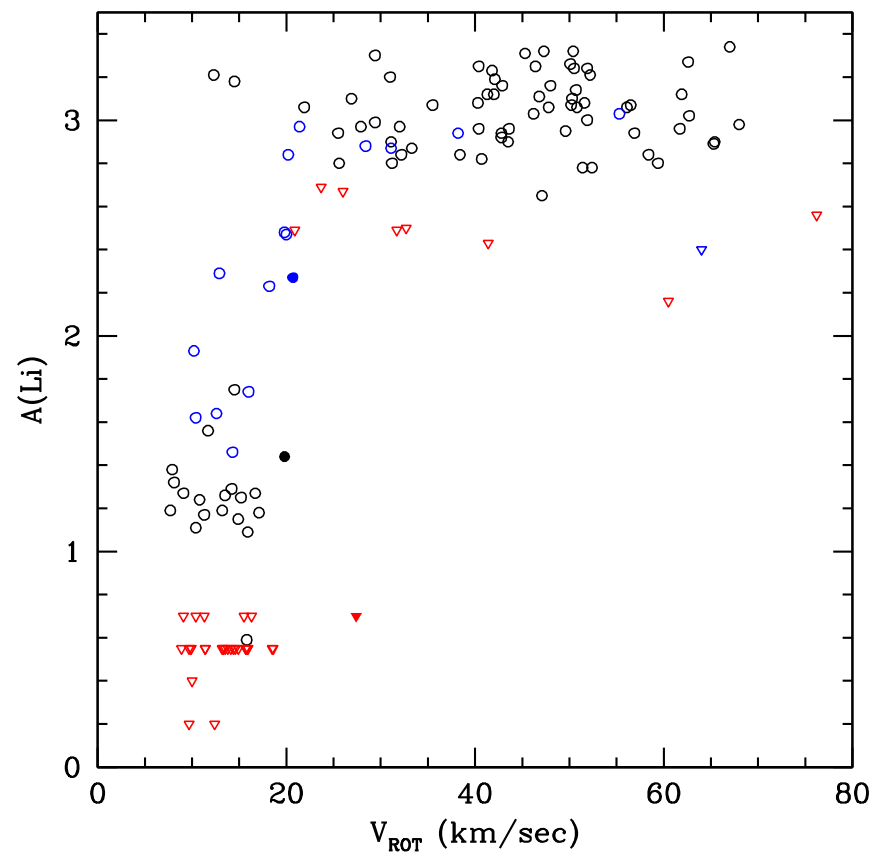

Figure 8. Li abundance as a function of rotational speed. Symbols have the same meaning as in Figure 5.

Li-dip temperature boundary is even more evident in the slightly older cluster, NGC 6819, where almost the entire turnoff region brighter than the Li-dip lies redward of the Li$\operatorname{dip} T_{\text {eff }}$ boundary but these higher-mass stars retain a range of A(Li) extending to 3.2 (C. P. Deliyannis et al. 2018, in preparation). Therefore, while $T_{\text {eff }}$ can play a valuable role in marking the boundary for the initiation of extra mixing and/or Li dilution not predicted by SSET for stars on or leaving the main sequence, it supplies no particular insight into the physical mechanism driving the process of depletion.

An alternative possibility emerges from the other significant stellar property which changes decisively for stars entering and crossing the subgiant branch, $V_{\text {rot }}$. Figure 8 shows the trend of $\mathrm{A}(\mathrm{Li})$ as a function of $V_{\text {rot }}$ for the stars used in Figures 5-7. Symbols have the same meaning as in the previous figures. First, there is no apparent correlation between $\mathrm{A}(\mathrm{Li})$ and rotational speed. As discussed earlier, the majority of stars for which reliable $\mathrm{A}(\mathrm{Li})$ estimation was deemed implausible were rapid rotators $\left(V_{\text {rot }}>30 \mathrm{~km} \mathrm{~s}^{-1}\right)$. A significant concern for such rotators is that the increased blending of the neighboring lines makes it challenging to judge the continuum level and account for line contamination. Unlike dwarfs in the Hyades and Praesepe (Cummings et al. 2017), however, a metallicity less than half that of the younger clusters makes this less of an issue and clearly introduces no trend in the data for stars where $\mathrm{A}(\mathrm{Li})$ is detectable.

Second, keeping in mind that the sin $i$ factor can move any star toward a lower than true rotation speed, i.e., to the left in Figure 8, the sharp transition near $V_{\text {rot }}=25 \mathrm{~km} \mathrm{~s}^{-1}$ is striking. Stars with $\mathrm{A}(\mathrm{Li})$ covering the range from 2.75 to 3.35 can have virtually any rotation speed from 12 to $65 \mathrm{~km} \mathrm{~s}^{-1}$; by contrast, with only one exception, every star with $\mathrm{A}(\mathrm{Li})$ below 2.4 has $V_{\text {rot }}$ below $25 \mathrm{~km} \mathrm{~s}^{-1}$.

The significance of the distribution of $\mathrm{A}(\mathrm{Li})$ with $V_{\text {rot }}$ among turnoff stars and giants becomes apparent when NGC 2506 is placed in the context of clusters ranging in age from $1.4 \mathrm{Gyr}$ (NGC 7789) to NGC 3680 (1.7 Gyr) to NGC 6819 (2.25 Gyr).
As detailed by C. P. Deliyannis et al. (2018, in preparation), for stars brighter than the Li-dip at the cluster turnoff, the range in $V_{\text {rot }}$ extends above $100 \mathrm{~km} \mathrm{~s}^{-1}$ for the youngest cluster, declines to $\sim 60 \mathrm{~km} \mathrm{~s}^{-1}$ for the intermediate-age cluster, and drops to $\sim 25 \mathrm{~km} \mathrm{~s}^{-1}$ for the oldest. NGC 2506 therefore most resembles NGC 3680, taking into account the lower mass of the Li-dip at lower $[\mathrm{Fe} / \mathrm{H}]$. Equally relevant, as the cluster age rises, the fraction of stars at the turnoff and more massive than the Li-dip stars with depleted or undetectable Li rises from less than $15 \%$ to more than $70 \%$. This trend also translates into a dramatic evolution of the Li among the giants. While more than half of the evolved stars in NGC 2506 have detectable Li, only seven of the 51 stars classed as subgiants or giants in NGC 6819 have detectable Li (C. P. Deliyannis et al. 2018, in preparation).

Before continuing with the distribution of $\mathrm{A}(\mathrm{Li})$ among the giants on the FRG branch, we can ask how well the empirical observations of Li evolution from the main sequence through the subgiant branch agree with the predictions of SSET, in the absence of two critically important processes, rotation-induced mixing and thermohaline mixing. The general answer is that, on many details, they do not agree. As first emphasized by Pasquini et al. (2004) using the models of Charbonnel \& Talon (1999) and Palacios et al. (2003) applied to the sparse data for IC 4651, models without rotation are incapable of explaining any decline in $\mathrm{A}(\mathrm{Li})$ among intermediate mass stars $(M=$ $1.8 M_{\odot}$ ) on the main sequence and beyond until the FDU, well across the Hertzsprung gap, in contradiction with the cluster data. By contrast, stellar models with the same mass but rotating above $100 \mathrm{~km} \mathrm{~s}^{-1}$ show significant depletions in atmospheric Li initiated at temperatures much hotter than in the non-rotating stars and comparable to the $T_{\text {eff }}$ of stars just leaving the main sequence.

The analysis of IC 4651 was revised using the newer models of Charbonnel \& Lagarde (2010) to include rotation-induced mixing, internal gravity waves, atomic diffusion, and thermohaline mixing and expanded to include the evolutionary pattern for $\mathrm{Be}$, once again confirming the need for additional mixing processes beyond simple convection to explain the abundance patterns observed in field stars and clusters (Smiljanic et al. 2010). With the exceptionally well-defined trend of $\mathrm{A}(\mathrm{Li})$ across the subgiant branch of NGC 2506, we can more effectively test the model predictions using the discussion of Charbonnel \& Lagarde (2010). The closest model analog to the stars at the turnoff and along the giant branch of NGC 2506 which have all three variants: standard evolution, standard with thermohaline mixing, and standard with thermohaline mixing and rotation are the $1.5 M_{\odot}$ models with rotation speeds near 0 or $110 \mathrm{~km} \mathrm{~s}^{-1}$. Note that the model metallicity is solar and the rotation speed is larger than observed for the stars in NGC 2506 , but our primary interest is in the qualitative pattern.

If we use standard evolution with or without thermohaline mixing, non-rotating stars leaving the main sequence retain their primordial $\mathrm{A}(\mathrm{Li})$ until they are almost $1600 \mathrm{~K}$ cooler than the turnoff $T_{\text {eff }}$, or $(B-V)=0.75$ for NGC 2506. At the hottest point of the turnoff, the surface convection zone (SCZ) occupies a tiny fraction by mass of the outermost layers. As the model evolves to cooler $T_{\text {eff }}$ along the subgiant branch, the SCZ deepens substantially and continuously. In standard theory (e.g., Deliyannis et al. 1990), the Li abundance as a function of depth has not been altered substantially while on the the main sequence until a depth is reached where $\mathrm{Li}$ is broken apart by 
energetic protons, the Li preservation boundary. Below that boundary, Li declines steeply with depth. Therefore, the surface Li abundance stays constant until the SCZ deepens sufficiently to reach the Li preservation boundary. As noted earlier, this happens at $T_{\text {eff }} \sim 5600 \mathrm{~K}$. Further evolution begins to reveal the impact of the FDU, as the SCZ deepens into regions where $\mathrm{Li}$ was destroyed during the main-sequence and pre-mainsequence phases and are thus now devoid of Li. Even as the SCZ deepens, the model expands and these layers are now much cooler than they were during the main sequence: no further Li destruction occurs at the base of the SCZ. Instead, convection mixes the outermost regions that still contain $\mathrm{Li}$ with those regions that are devoid of $\mathrm{Li}$, resulting in a decrease of the surface Li abundance, i.e., subgiant dilution.

This decrease is a steep function of decreasing $T_{\text {eff }}$ because, by the time the SCZ reaches the base of the FRG branch, it includes more than half of the stellar mass. By contrast, the $\mathrm{Li}$ preservation region occupies only a small fraction of the stellar mass, so the total dilution of surface Li is roughly $1.8 \mathrm{dex}$ (Deliyannis et al. 1990; Charbonnel \& Lagarde 2010). The SCZ reaches a maximum depth near the base of the FRG branch at $\sim 4800 \mathrm{~K}$, after which the base of the SCZ recedes slowly toward the surface, as the model evolves up the FRG branch. Standard theory predicts no further depletion of surface Li up the giant branch, thereby establishing the subgiant diluted Li plateau, namely a constant $\mathrm{Li}$ abundance up the FRG. If the turnoff $\mathrm{A}(\mathrm{Li}) \sim 3.3$, then the diluted plateau is predicted to have $\mathrm{A}(\mathrm{Li}) \sim 1.5$. If $\mathrm{A}(\mathrm{Li})$ of the star leaving the turnoff is lower, either due to a lower primordial $\mathrm{Li}$ value associated with a lower metallicity cluster and/or due to non-standard $\mathrm{Li}$ depletion on the main sequence, then the diluted plateau $\mathrm{A}(\mathrm{Li})$ could easily approach $\mathrm{A}(\mathrm{Li})=1.2$. This would be consistent with the plateau of Li abundances seen in Figures 6 and 7. But what about those stars with $\mathrm{A}(\mathrm{Li})$ lying substantially below the diluted Li plateau?

Subgiant evolution can show the effects of non-standard surface Li depletion mechanisms that might have acted during the main sequence and beyond, and that might have reduced the amount of $\mathrm{Li}$ in the $\mathrm{Li}$ preservation region. In the case of the models of Charbonnel \& Lagarde (2010), inclusion of rotation and rotationally induced mixing produces an immediate and continuous decline in $\mathrm{A}(\mathrm{Li})$ as soon as the star begins evolving away from the main sequence toward the giant branch. By the end of the FDU phase the atmospheric $\mathrm{A}(\mathrm{Li})$ is reduced to less than 0.5 . It is expected that adoption of a lower initial rotation rate would generate the same trend, but approach a higher limit for $\mathrm{A}(\mathrm{Li})$ beyond the FDU. Exactly how the final value for $\mathrm{A}(\mathrm{Li})$ correlates with increased $V_{\text {rot }}$ requires extensive modeling beyond those currently available.

Moving beyond the base of the FRG branch, the deficiency in $\mathrm{Li}$ among the clump stars is predictable since all these stars have supposedly undergone He-flash, generating mixing which could reduce and/or eliminate any remaining signs of the element in their atmospheres as they arrive at the red giant tip. While burning $\mathrm{He}$ in a stable configuration on the clump, the $\mathrm{A}(\mathrm{Li})$ should remain unchanged until the star begins its ascent up the asymptotic giant branch and approaches the second dredge-up phase.

A more uncertain interpretation applies to the concentration of Li-deficient stars at the base of the giant branch. The key structural feature which kicks in near this phase of evolution is the FDU. As already noted, if the SCZ reaches deep enough, it

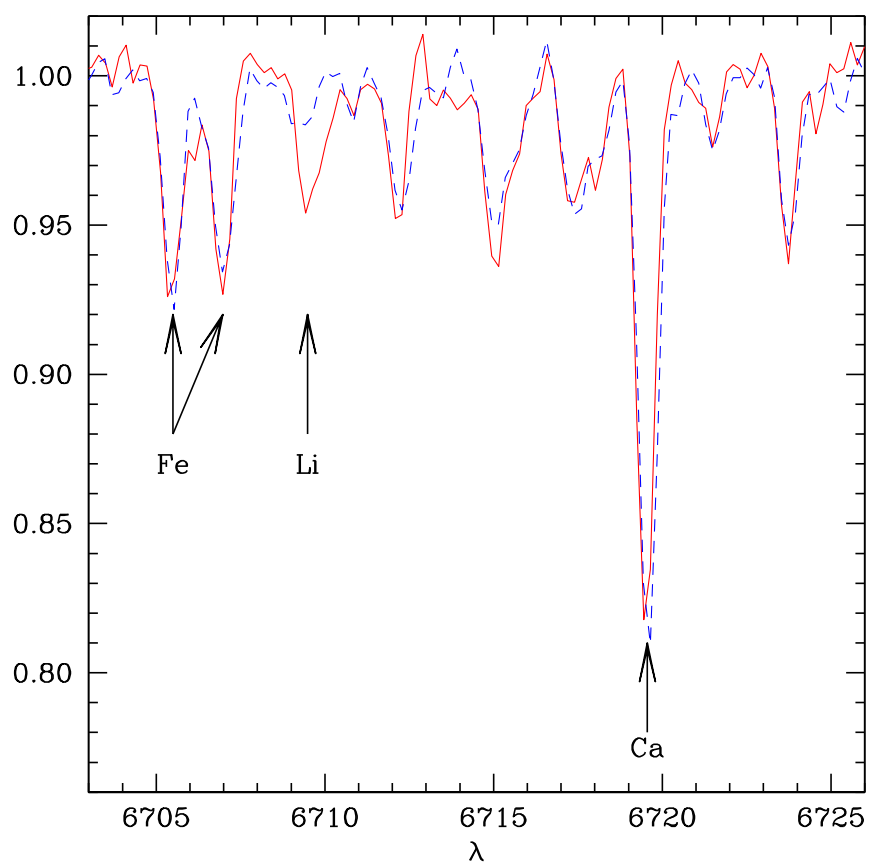

Figure 9. Closeup of the lithium line region in two stars at the base of the giant branch. The red spectrum traces star 1301, the blue spectrum star 7008. A number of lines in the Li region are identified.

can mix atmospheric Li with Li-depleted layers well below the Li-preservation zone, potentially causing a sudden and significant decrease in the observed $\mathrm{A}(\mathrm{Li})$, as seen in Figure 5 near $V=14.8$. If this is the case, why then do the majority of stars on the FRG branch between $V=14.6$ and 13 in Figure 5 have measurable and constant $\mathrm{A}(\mathrm{Li})$ ?

One potential solution is that the difference between measurable and detectable $\mathrm{Li}$ among the spectra is small enough that only a slight change in the strength of the line will shift a star from one category to the other, i.e., the bimodal $\mathrm{Li}$ distribution among these giants is an artifact of the spectra. To illustrate why this fails, we show in Figure 9 a comparison of two stars at the base of the giant branch with virtually identical colors and magnitudes, star 1301 with $\mathrm{A}(\mathrm{Li})$ near 1.56 and star 7008 with only an upper limit near $\mathrm{A}(\mathrm{Li})=0.6$. It is clear that there is a distinct difference in the strength of the Li line for these two stars, consistent with their classification, even though all other lines have the same strength within the spectroscopic uncertainties.

Assuming that the majority of these stars are members and do not represent a low-luminosity extension of the red giant clump, two plausible options exist: (a) these stars are not single stars, but instead are evolved binaries/blue stragglers approaching the base of the giant branch at a higher luminosity, or (b) the stars with more extreme Li-depletion than the Liplateau stars are those which initially had the highest rotation speeds and have been systematically more affected by the mixing processes controlled by rotation and stellar spindown. We reiterate that one of the stars among this group has a spectroscopic $[\mathrm{Fe} / \mathrm{H}]$ near solar indicating that, despite the radial velocity agreement with the cluster value, it may be a field interloper.

From Figure 7, among the stars with measurable $\mathrm{Li}$, there is little evidence for a decline in $\mathrm{A}(\mathrm{Li})$ as one moves up the giant branch to the level of the clump. Instead, from 16 FRGs (open circles in Figures 5-7), the stars have $\mathrm{A}(\mathrm{Li})=1.25$ with a 
scatter of \pm 0.11 dex. If one star near the base of the FRG is excluded, the average becomes $1.22 \pm 0.08$, only slightly higher than expected from the precision of the measures, but significantly lower than found among the stars at the turnoff. For SSET and for models with rotational mixing on the main sequence, $\mathrm{A}(\mathrm{Li})$ for a star is predicted to remain constant at the value it has attained after completion of the FDU.

The next key change predicted in the surface A(Li) occurs once the stars pass beyond the red giant bump. The latter represents an evolutionary slowdown and reversal as the hydrogen-burning shell passes across the chemical composition discontinuity created by the high-water mark of the convection zone as the stars evolved toward and up the giant branch. The change in molecular weight and $\mathrm{H}$ concentration allows the giant to support itself at a lower luminosity and then evolve back up the giant branch at a slower rate, causing a density excess in the distribution of giants. Standard models predict that stars evolving beyond the bump will experience no change in $\mathrm{A}(\mathrm{Li})$ until they reach the tip of the giant branch. By contrast, if one includes thermohaline mixing, stars brighter than the bump will decrease their lithium by 0.4 dex. The location of the red giant bump in NGC 2506, predicted by VR isochrones with age between 1.8 and $1.9 \mathrm{Gyr}$ and $[\mathrm{Fe} / \mathrm{H}]=$ -0.29 , is between $V=12.95$ and 12.7 , above the observed level of the clump. Clearly, there is no excess concentration of stars in this magnitude range in Figure 5. However, the Charbonnel \& Lagarde (2010) models for the $1.5 M_{\odot}$ with rotation $\left(V_{\text {rot }}=110 \mathrm{~km} \mathrm{~s}^{-1}\right)$ demonstrate that the presence of rotation will shift the location of the red giant bump and the start of thermohaline mixing to a lower luminosity on the giant branch, typically $40 \%$ fainter for the bump and a factor of almost 8 for the mixing trigger, placing the mixing phase only 3-4 times brighter than the red giant bump. While the size of the change will depend upon the specific size of the rotation, inclusion of rotation should move the bump fainter than the level of the red giant clump. Equally important, additional depletion of Li caused by thermohaline mixing should begin to show among stars on the FRG branch no more than a magnitude brighter than the clump. Thus, the redefinition of the FRG branch passing through star 2402 with detectable Li just below $\mathrm{A}(\mathrm{Li})=0.6$ is consistent with a significant decline from $\mathrm{A}(\mathrm{Li})=1.25$ caused by mixing in a star beyond the red giant bump. The other stars brighter than 2402 at comparable colors would then be either binaries undergoing peculiar evolution or stars ascending the asymptotic giant branch.

\section{Summary and Conclusions}

HYDRA spectroscopy of 287 stars in the field of NGC 2506 has been used in conjunction with published proper-motion membership and position in the CMD to identify highly probable, single-star members of the cluster for further analysis. The survey included 24 stars within the previously studied area of the cluster and 135 stars outside the cluster area for which the only available information was the location in the CMD. Of these 159, 94 proved to be probable radial-velocity members, adding a critical number of stars to evolutionary phases of the CMD which are normally poorly, if at all, populated. Abundance analysis for metallicity determination, dominated by the richer and stronger line profiles of the cooler stars, confirms that NGC 2506 is moderately metal-poor, with $[\mathrm{Fe} / \mathrm{H}]$ near -0.3 .
Returning to the primary focus of the investigation, the evolution of $\mathrm{Li}$ within the cluster as a function of mass, a number of conclusions stand out.

There is an intrinsic spread in $\mathrm{A}(\mathrm{Li})$ among the stars at the vertical turnoff, though the range is independent of stellar luminosity or color. Since all the observed turnoff stars lie brighter than the Li-dip and, by SSET, have retained their initial $\mathrm{Li}$ abundance unchanged by evolution, the mean $\mathrm{A}(\mathrm{Li})$ for the cluster is calculated to be $3.05 \pm 0.16$, consistent with a lower initial abundance than the Sun (Asplund et al. 2009), as predicted for a cluster which is a factor of two below solar in $[\mathrm{Fe} / \mathrm{H}]$. It is noted, however, that the full range of the sample in $\mathrm{A}(\mathrm{Li})$ extends from 2.75 to 3.35 , comparable to that found among stars blueward of the Li-dip in clusters of solar metallicity or higher.

Upon exiting the turnoff region and evolving across the subgiant branch, the stars undergo a well-delineated decline in atmospheric $\mathrm{Li}$, declining from a mean of $\mathrm{A}(\mathrm{Li})=3.05$ to a plateau value of 1.25 by the base of the FRG branch. While it has been obvious for decades that the typical red giant contains less $\mathrm{Li}$ than expected for a star of the same mass on the main sequence, it has been a challenge to identify precisely when and where the depletion occurs. Stars fainter than the clump in clusters of intermediate age exhibit depleted $\mathrm{Li}$, but the degree of depletion can vary by a factor of 10 and it can be difficult to assess if the star is truly a first-ascent or red clump giant.

We reiterate that the evolutionary phase under discussion is not the vertical turnoff region. Intermediate-age clusters like NGC 3680 and NGC 752 do show a spread in A(Li) ranging from 3.3 to 2.6 at the top of the vertical turnoff (AnthonyTwarog et al. 2009), virtually identical to the range found in NGC 2506. IC 4651 exhibits the same pattern, but is the one cluster in the 1-2 Gyr age category to include multiple stars populating the subgiant branch between the vertical turnoff and the red giant branch. Pasquini et al. (2004) have studied a limited sample of stars in IC 4651 and find four at the top of the turnoff and one at a temperature intermediate between the turnoff stars and the red clump which have $\mathrm{A}(\mathrm{Li})$ between 2.4 and 1.6. Note that the one star with a $T_{\text {eff }}$ placing it in the subgiant region has $\mathrm{A}(\mathrm{Li})=2.1$. For the first time, the mapping of a subgiant branch using stars outside the Li-dip demonstrates that these stars continuously deplete Li as they evolve to the base of the FRG branch, irrespective of whatever mechanism, if any, produces the dispersion among stars at the top of the turnoff.

A contributing factor leading to the onset Li-depletion on the subgiant branch appears to be the spindown of the stars as they evolve to cooler temperatures at almost constant luminosity. Stars at the vertical turnoff within the $\mathrm{A}(\mathrm{Li})$ range discussed earlier can have any rotation speed between $\sim 10 \mathrm{~km} \mathrm{~s}^{-1}$ and $70 \mathrm{~km} \mathrm{~s}^{-1}$, with the caveat that the true rotation speed could be higher and that the spectra have resolution which limits the $V_{\text {rot }}$ to a minimum approaching $10 \mathrm{~km} \mathrm{~s}^{-1}$. There is no correlation between luminosity and/or temperature and $V_{\text {rot }}$. By contrast, as stars evolve across the subgiant branch and the typical $V_{\text {rot }}$ declines from a minimum of $25 \mathrm{~km} \mathrm{~s}^{-1}$ to an average near $13 \mathrm{~km} \mathrm{~s}^{-1}, \mathrm{~A}(\mathrm{Li})$ drops steadily to 1.25 . This pattern fits perfectly within the trend defined by NGC 7789, NGC 3680, and NGC 6819, where the fraction of turnoff and giant stars with detectable Li is strongly correlated with the spread in rotation speed among stars hotter than the Li-dip; the larger the range in speed at a given age, the more likely the turnoff stars 
will have a modest range in $\mathrm{A}(\mathrm{Li})$ with a mean at or above 3.0, and the more likely that the giants will exhibit detectable Li near 1 (C. P. Deliyannis et al. 2018, in preparation).

It should be noted that the $V_{\text {rot }}$ distribution for stars brighter than the Li-dip at the turnoff and on the giant branch of IC 4651 (Meibom et al. 2002) is virtually identical to that in NGC 3680 . For stars at the turnoff, the range is from $\sim 10 \mathrm{~km} \mathrm{~s}^{-1}$ to more than $60 \mathrm{~km} \mathrm{~s}^{-1}$ while the giants exhibit a small scatter near $1 \mathrm{~km} \mathrm{~s}^{-1}$ (Meibom et al. 2002). The uncertainty in the latter velocity measures is analogous to that for Carlberg (2014) rather than our HYDRA data. Unfortunately, when binaries are eliminated, the current number of stars with reliable $\mathrm{Li}$ determinations brighter than the Li-dip is too small to shed any statistical light on the question. A comprehensive survey of this cluster with a significant expansion of the spectroscopic sample could prove invaluable.

The Li pattern exhibited at the turnoff is consistent with the predictions of stellar models of low/intermediate mass which include rotation-induced mixing on the main sequence and beyond (Charbonnel \& Lagarde 2010). The growing dispersion in $\mathrm{Li}$ for stars on the blue side of the Li-dip as clusters age from 1 to 2 Gyrs then becomes a reflection of the initial spread in $V_{\text {rot }}$ acting over time to reduce the absolute $\mathrm{Li}$ at Hyades age from a uniform value at or above $\mathrm{A}(\mathrm{Li})=3.0$. This dispersion is then coupled with the significant spindown of all the stars evolving along the subgiant branch, reducing the $\mathrm{Li}$ abundance to a typical value of 1.2 by the base of the FRG and the end of the FDU. If the decline in $\mathrm{A}(\mathrm{Li})$ at the start of the subgiant branch is not tied to the evolution of the rotation rate but simply defines the start of the FDU, this phase is triggered at a much higher $T_{\text {eff }}(6450 \mathrm{~K})$ than predicted by the non-rotating models $(5600 \mathrm{~K})$.

A fundamental challenge to this qualitative explanation for the spread in $\mathrm{A}(\mathrm{Li})$ among the turnoff stars is presented by the absence of a comparable spread among the evolved stars of the FRG branch. The dispersion in $\mathrm{A}(\mathrm{Li})$ for stars at the turnoff is twice that among the giants; if stars leave the main sequence with a factor of 4 range in the $\mathrm{Li}$ abundance due to the range in $V_{\text {rot }}$, why does that spread not persist among stars on the FRG branch? An alternative solution to the dispersion question is presented by diffusion among the metal-deficient stars at the cluster turnoff. For very thin SCZs among stars in the $T_{\text {eff }}$ range $6800-7100 \mathrm{~K}, \mathrm{Li}$ can undergo radiative acceleration into the SCZ, creating potentially significant surface overabundances, if the radiative acceleration region is much larger by mass fraction than the SCZ (Richer \& Michaud 1993). If the initial $\mathrm{A}(\mathrm{Li})$ for the metal-poor NGC 2506 was $\sim 3.0$, this mechanism could create surface $\mathrm{A}(\mathrm{Li})$ as high as 3.3 or higher, without destroying $\mathrm{Li}$ inside the star. Inside the star, below the radiative acceleration boundary, Li sinks, with a small amount of it potentially reaching depths where some, but not much, might be destroyed. For stars cooler than about $6700 \mathrm{~K}$, the radiative acceleration boundary is within the SCZ, so only downward diffusion at the base of the SCZ can occur. The net result is that as stars leave the main sequence and the SCZ grows, mixing of the inhomogeneous layers returns $\mathrm{A}(\mathrm{Li})$ to the initial value of $\sim 3.0$ with only a modest dispersion. Beyond that point, whatever mechanisms exist to deplete $\mathrm{Li}$ on the subgiant branch and beyond act on a significantly more homogeneous initial abundance. Clearly, deciding on the relative viability of either process is beyond the scope of the current observations.
Moving to the FRG branch, it is tempting to associate the handful of Li-deficient giants at the base of the FRG with the FDU as the SCZ accesses deeper and hotter layers below the atmosphere. If the FDU is being outlined by the stars with upper limits to $\mathrm{Li}$, it would be beneficial to look for other signatures of mixed processed nuclear material, e.g., ${ }^{12} \mathrm{C} /{ }^{13} \mathrm{C}$ ratios, in the atmospheres of these and the more evolved stars (e.g., Charbonnel \& Lagarde 2010; Karakas \& Lattanzio 2014). To date, ${ }^{12} \mathrm{C} /{ }^{13} \mathrm{C}$ ratios have been measured for three giants by Mikolaitis et al. (2011) and three single-star (3265 excluded) giants by Carlberg et al. (2016). Five of the six stars are located in the clump and one sits 1.5 mag above the clump, classified as a red-giant-tip star by Mikolaitis et al. (2011), though this becomes debatable in light of the CMD position of 2402 . Ignoring for now the one star with a lower limit to the ${ }^{12} \mathrm{C} /{ }^{13} \mathrm{C}$ ratio of 10 , the remaining five stars have an average ratio of $10 \pm 2$, indicating that they all have the same ${ }^{12} \mathrm{C} /{ }^{13} \mathrm{C}$ ratio, within the uncertainties for the individual measures. This low value is also consistent with rotating models which include thermohaline mixing, whether the star is at the red giant tip or completing the second dredge-up phase (Charbonnel \& Lagarde 2010). The models do predict a significantly higher value above 20 for stars completing the FDU. So, while the ${ }^{12} \mathrm{C} /{ }^{13} \mathrm{C}$ ratio cannot distinguish between a first- or secondascent red giant, it should provide insight into the status of the Li-depleted giants at the base of the FRG.

For stars with detectable Li on the FRG, within the scatter, there appears to be no evidence for a systematic depletion of $\mathrm{Li}$ up to the level of the red clump. The one star (2402) above the clump with detectable $\mathrm{Li}$, if it defines the path of the FRG above the clump, exhibits depletion relative to the other giants which is consistent with a post-bump giant undergoing thermohaline mixing. The stars within the clump, with one anomalously blue exception, exhibit only upper limits below the level of star 2402. For comparison, with four measured clump stars at higher resolution, Carlberg et al. (2016) determine a detectable LTE Li abundance (the appropriate comparison for our data) for two stars and an upper limit for two more. The average of the two stars with measured $\mathrm{Li}$ is $\mathrm{A}(\mathrm{Li})=0.63$ from individual values with $\sigma_{\mathrm{Li}}=0.14$. The two stars with only upper limits have average limits of $\mathrm{A}(\mathrm{Li})=0.45$. These estimates are clearly below 1.25 and totally consistent with our clump determinations obtained at lower resolution.

The authors are indebted to the referee for a thorough and invaluable reading of the original maunscript, identifying a number of potential points of confusion and a significant error in one of our equations. The paper has definitely been improved as a result. The authors gratefully acknowledge extensive use of the $\mathrm{WEBDA}^{8}$ database, maintained at the University of Brno by E Paunzen, C Stutz and J Janik. We also express appreciation to Jeffrey Cummings for sharing spectroscopic data with us. Support was provided to B.J.A.T., D.B.L. B. and B.A.T. through NSF grant AST-1211621 and to C.P.D. through AST-1211699.

Facility: WIYN:3.5m.

Software: ANNA (D. B. Lee-Brown 2018, in preparation; Lee-Brown 2017), IRAF, ROBOSPECT (Waters \& Hollek 2013), SPECTRUM (Gray \& Corbally 1994).

\footnotetext{
http://webda.physics.muni.cz
} 


\section{ORCID iDs}

Barbara J. Anthony-Twarog (i) https://orcid.org/0000-00018841-3579

Donald B. Lee-Brown (ib https://orcid.org/0000-00030164-0600

\section{References}

Ahn, C. P., Alexandroff, R., Allende Prieto, C., et al. 2014, ApJS, 211, 17 Allende Prieto, C., Rebolo, R., García López, R. J., et al. 2000, AJ, 120, 1516 Anthony-Twarog, B. J., Atwell, J., \& Twarog, B. A. 2005, AJ, 129, 872 Anthony-Twarog, B. J., Deliyannis, C. P., \& Twarog, B. A. 2016, AJ, 152, 192 Anthony-Twarog, B. J., Deliyannis, C. P., Twarog, B. A., Croxall, K. V., \& Cummings, J. 2009, AJ, 138, 1171

Anthony-Twarog, B. J., \& Twarog, B. A. 2000, AJ, 119, 2282

Arbib, M. A. 2002, The Handbook of Brain Theory and Neural Networks (2nd ed.; Cambridge, MA: MIT Press)

Arentoft, T., De Ridder, J., Grundahl, F., et al. 2007, A\&A, 465, 965

Asplund, M., Grevasse, N., Sauval, A. J., \& Scott, P. 2009, ARA\&A, 47, 481

Bailer-Jones, C. A. L., Irwin, M., Gilmore, G., \& von Hippel, T. 1997, MNRAS, 292, 157

Balachandran, S. 1995, ApJ, 446, 203

Balachandran, S., Anthony-Twarog, B. J., \& Twarog, B. A. 1991, in ASP Conf. Ser. 13, The Formation and Evolution of Star Clusters, ed. K. Janes (San Francisco, CA: ASP), 544

Balachandran, S. C., Mallik, S., \& Lambert, D. L. 2010, MNRAS, 410, 2526

Bruntt, H., Basu, S., Smalley, B., et al. 2012, MNRAS, 423, 122

Carlberg, J. K. 2014, AJ, 147, 138

Carlberg, J. K., Cunha, K., \& Smith, V. V. 2016, ApJ, 827, 129

Carretta, E., Bragaglia, A., Gratton, R. G., \& Tosi, M. 2004, A\&A, 422, 951

Casey, A. R., Ruchti, G., Masseron, T., et al. 2016, MNRAS, 461, 3336

Charbonnel, C., \& Lagarde, N. 2010, A\&A, 522, 10

Charbonnel, C., \& Talon, S. 1999, A\&A, 351, 635

Chen, Y. Q., Nissen, P. E., Benoni, T., \& Zhao, G. 2001, A\&A, 371, 943

Chiu, L.-T. G., \& van Altena, W. F. 1981, ApJ, 243, 827

Coc, A., Uzan, J.-P., \& Vangioni, E. 2014, JCAP, 10, 50

Cortés, C., Silva, J. R. P., Recio-Blanco, A., et al. 2009, ApJ, 704, 750

Cummings, J. D. 2011, PhD thesis, Univ. Indiana

Cummings, J. D., Deliyannis, C. P., Anthony-Twarog, B. J., Twarog, B. A., \& Maderak, R. M. 2012, AJ, 144, 137

Cummings, J. D., Deliyannis, C. P., Maderak, R. M., \& Steinhauer, A. 2017, AJ, 153, 128

Dafonte, C., Fustes, D., Manteiga, M., et al. 2016, A\&A, 594, A68

Deliyannis, C. P., Demarque, P., \& Kawaler, S. D. 1990, ApJS, 73, 21

Deliyannis, C. P., \& Pinsonneault, M. H. 1993, in ASP Conf. Ser. 40, IAU Coll. 137, Inside the Stars, ed. W. W. Weiss \& A. Baglin (San Francisco, CA: ASP), 174

Deliyannis, C. P., Pinsonneault, M. H., \& Duncan, D. K. 1993, ApJ, 414, 740 Deliyannis, C. P., Steinhauer, A., \& Jeffries, R. D. 2002, ApJL, 577, L39

Dobrovolskas, V., Kučinskas, A., Bonifacio, P., et al. 2014, A\&A, 565, 121

Edvardsson, B., Andersen, J., Gustafsson, B., et al. 1993, A\&A, 275, 101

Francois, P., Pasquini, L., Biazzo, K., Bonifacio, P., \& Palsa, R. 2013, A\&A, 552, 136

Friel, E. D., \& Janes, K. A. 1993, A\&A, 267, 75

Friel, E. D., Janes, K. A., Tavarez, M., et al. 2002, AJ, 124, 2693

Fu, X., Romano, D., Bragaglia, A., et al. 2017, arXiv:1711.04829

Gray, R. O., \& Corbally, C. J. 1994, AJ, 107, 742
Hill, V., \& Pasquini, L. 2000, in IAU Symp. 198, The Light Elements and Their Evolution, ed. L. da Silva, R. de Medeiros, \& M. Spite (San Francisco, CA: ASP), 293

Jacobson, H. R., Friel, E. D., \& Pilachowski, C. A. 2011, AJ, 141, 58

Jones, B. F., Fischer, D., Shetrone, M., \& Soderblom, D. R. 1997, AJ, 114, 352 Karakas, A. I., \& Lattanzio, J. C. 2014, PASA, 31, 30

Kim, S.-L., Chun, M.-Y., Park, B.-G., et al. 2001, AcA, 51, 49

Kumar, Y. B., Reddy, B. E., \& Lambert, D. L. 2011, ApJL, 730, 12

Kupka, F., Piskunov, N., Ryabchikova, T. A., Stempels, H. C., \& Weiss, W. W. 1999, A\&AS, 138, 119

Kurucz, R. L. 1992, in IAU Symp. 149, The Stellar Populations of Galaxies, ed. B. Barbuy \& A. Renzini (Dordrecht: Kluwer), 225

Landolt, A. U. 1992, AJ, 104, 340

Lee, S. H., Kang, Y.-W., \& Ann, H. B. 2012, MNRAS, 425, 1567

Lee, S. H., Kang, Y.-W., \& Ann, H. B. 2013, MNRAS, 432, 1672

Lee-Brown, D. B. 2017, Artificial Neural Network Abundances (ANNA), v0.1.0, Zenodo, doi:10.5281/zenodo.1043585

Lee-Brown, D. B., Anthony-Twarog, B. J., Deliyannis, C. P., Rich, E., \& Twarog, B. A. 2015, AJ, 149, 121

Li, X.-R., Pan, R.-Y., \& Duan, F.-Q. 2017, RAA, 17, 36

Manteiga, M., Ordóñez, D., Dafonte, C., \& Arcay, B. 2010, PASP, 122, 608

Marconi, G., Hamilton, D., Tosi, M., \& Bragaglia, A. 1997, MNRAS, 291, 763

McClure, R. D., Twarog, B. A., \& Forrester, W. T. 1981, ApJ, 243, 841

Meibom, S., Andersen, J., \& Nordström, B. 2002, A\&A, 386, 187

Mermilliod, J.-C., \& Mayor, M. 2007, A\&A, 470, 919

Mermilliod, J.-C., Mayor, M., \& Udry, S. 2008, A\&A, 485, 303

Mikolaitis, S., Tautvaišiene, G., Gratton, R., Bragaglia, A., \& Carretta, E. 2011, MNRAS, 416, 1092

Minniti, D. 1995, A\&AS, 113, 299

Monaco, L., Boffin, H. M. J., Bonifacio, P., et al. 2014, A\&A, 564, L6

Netopil, M., Paunzen, E., Heiter, U., \& Soubiran, C. 2016, A\&A, 585, 150

Palacios, A., Talon, S., Charbonnel, C., \& Forestini, M. 2003, A\&A, 399, 603

Palmerini, S., Cristallo, S., Busso, M., et al. 2011, ApJ, 741, 26

Pasquini, L., Randich, S., Zoccali, M., et al. 2004, A\&A, 424, 951

Pinsonneault, M. 1997, ARA\&A, 35, 557

Ramírez, I., Allende Prieto, C., \& Lambert, D. L. 2013, ApJ, 764, 78

Ramírez, I., Fish, J. R., Lambert, D. L., \& Allende Prieto, C. 2012, ApJ, 756, 46

Ramírez, I., \& Meléndez, J. 2005, ApJ, 626, 465

Randich, S., Pace, G., Pastori, L., \& Bragaglia, A. 2009, A\&A, 496, 441

Reddy, A. B. S., Giridhar, S., \& Lambert, D. L. 2012, MNRAS, 419, 1350

Richer, J., \& Michaud, G. 1993, ApJ, 416, 312

Santos, N. C., Lovis, C., Pace, G., Meléndez, J., \& Naef, D. 2009, A\&A, 493, 309

Silva Aguirre, V., Ruchti, G. R., Hekker, S., et al. 2014, ApJL, 784, 16

Smiljanic, R., Pasquini, L., Charbonnel, C., \& Lagarde, N. 2010, A\&A, 510,50

Sneden, C. 1973, ApJ, 184, 839

Snider, D., Allende Prieto, C., von Hippel, T., et al. 2001, ApJ, 562, 528

Steinhauer, A. 2003, PhD thesis, Indiana Univ.

Steinhauer, A., \& Deliyannis, C. P. 2004, ApJL, 614, L65

Stetson, P. B. 1987, PASP, 99, 191

Takeda, Y., \& Tajitsu, A. 2017, PASJ, 69, 74

Twarog, B. A., Anthony-Twarog, B. J., Deliyannis, C. P., \& Thomas, D. T. 2015, AJ, 150, 134

Twarog, B. A., Ashman, K., \& Anthony-Twarog, B. J. 1997, AJ, 114, 2556

Van Stockum, P., Matson, R., Deliyannis, C., Sarajedini, A., \& Platais, I. 2005, BAAS, 37, 71.06

VandenBerg, D. A., Bergbusch, P. A., \& Dowler, P. D. 2006, ApJS, 162, 375

Waters, C. Z., \& Hollek, J. K. 2013, PASP, 125, 1164 